
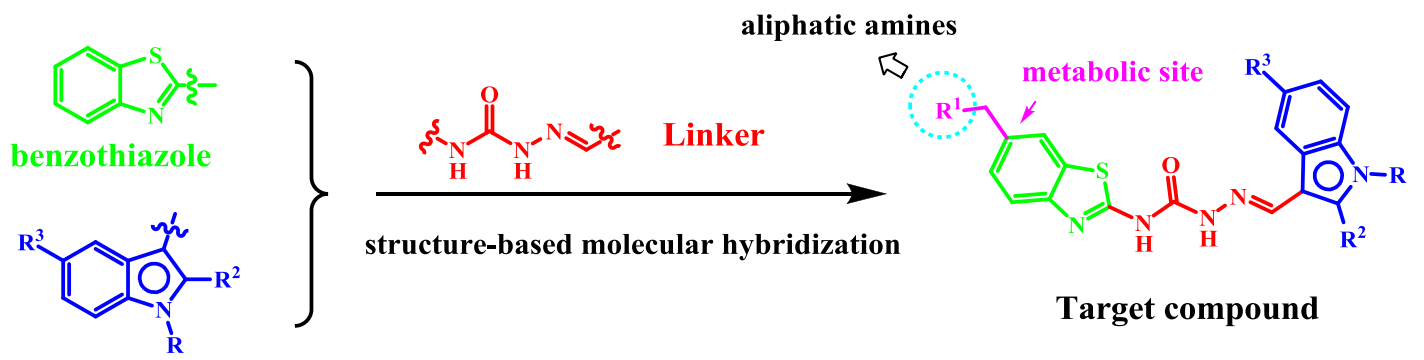

indole-based moiety

A novel series of benzothiazole derivatives bearing indole-based moiety were synthesized and evaluated for their cytotoxicity, enzymatic assays, cell cycle analysis and 3D-QSAR analysis. 


\title{
Design, synthesis, biological evaluation and preliminary mechanism study of novel benzothiazole derivatives bearing indole-based moiety as potent antitumor agents
}

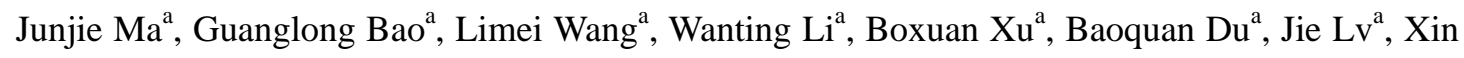
Zhai $^{\mathrm{a}, *}$, Ping Gong ${ }^{\mathrm{a}, * * *}$

${ }^{\mathrm{a}}$ Key Laboratory of Structure-Based Drug Design and Discovery (Shenyang Pharmaceutical University), Ministry of Education, 103 Wenhua Road, Shenhe District, 110016, P. R. China.

${ }^{*}$ Corresponding author. Tel./Fax: +86 242398 6429; E-mail address: leixinjia@126.com

** Corresponding author. Tel./Fax: +86 242398 6429; E-mail address: gongpinggp@ 126.com

\begin{abstract}
Through a structure-based molecular hybridization approach, a series of novel benzothiazole derivatives bearing indole-based moiety were designed, synthesized and screened for in vitro antitumor activity against four cancer cell lines (HT29, H460, A549 and MDA-MB-231). Most of them showed moderate to excellent activity against all the tested cell lines. Among them, compounds 20a-w with substituted benzyl- $1 H$-indole moiety showed better selectivity against HT29 cancer cell line than other compounds. Compound 20d exhibited excellent antitumor activity with $\mathrm{IC}_{50}$ values of $0.024,0.29,0.84$ and $0.88 \mu \mathrm{M}$ against HT29, H460, A549 and MDA-MB-231, respectively. Further mechanism studies indicated that the marked pharmacological activity of compound 20d might be ascribed to activation of procaspase-3 (apoptosis-inducing) and cell cycle arrest, which had emerged as a lead for further structural modifications. Furthermore, 3D-QSAR model (training set: $\mathrm{q}^{2}=0.850, \mathrm{r}^{2}=0.987$, test set: $\mathrm{r}^{2}=0.811$ ) was built to provide a comprehensive guide for further structural modification and optimization.
\end{abstract}

Keywords: synthesis; benzothiazole; indole-based moiety; 3D-QSAR; antitumor activity

\section{Introduction}

Although much important progress has been made in recent years, cancer remains the leading cause of death worldwide. It is expected that annual cancer cases will rise from 14 million in 2012 to 22 million within the next two decades [1]. Therefore, the discovery of more effective and safer antitumor agents remains urgently needed.

Indole ring system as one of the most ubiquitous heterocycles in nature, has been becoming an important structural component in many pharmaceutical agents, such as antidepressant[2], anticonvulsant[3], antifungal[4], antiviral[5] and anti-inflammatory[6], particularly in discovery of new antitumor agents[7,8]. In recent years, increasing numbers of researchers from both industry and academia have embarked on the development of new indole-based small molecules as potent antitumor agents [9,10]. For example, small molecule Bcl-2 antagonist obatoclax 1 (phase III, Fig. 1) could antagonize MCL-1 and overcome MCL-1-mediated resistance to apoptosis [11]. Sunitinib 2 (Fig. 1), a multi-targeted receptor tyrosine kinase inhibitor containing indolin-2-one moiety, was approved by the FDA for the treatment of advanced renal cell carcinoma (RCC) and gastrointestinal stromal tumors (GIST)[12]. Moreover, benzyl-1H-indole derivatives also possess prominent antitumor activity [13], such as a novel synthetic microtubule inhibitor D-24851 3 (Phase I/II, Fig. 1)[14] and oncrasin-1 4 (Fig.1), a cell-permeable 
indole-carboxaldehyde compound that could selectively induce apoptosis in cells harboring mutant K-Ras both in vitro[15].

(Fig. 1. should be listed here)

In our previous study, inspired by diverse biological properties of benzothiazole 5 (Fig. 2) [16-20], a series of derivatives containing the molecular skeleton of benzothiazole and ortho-hydroxy carbamoyl hydrazone moiety had been reported as antitumor agents 6 (Fig. 2)[21] . In addition, semicarbazone (-NH-CO-NH-N=CH-) was widely used as building blocks in design of different potential antitumor agents due to the presence of several hydrogen donors and acceptors as well as its flexible skeleton. As a result, several small molecule kinase inhibitors bearing semicarbazone had also been independently reported in our laboratory, e.g. compounds $\mathbf{7}$, 8 and 9 (Fig. 2), and indicated that introduction of semicarbazone as a linker was an effective approach for developing more potent and broad spectrum antitumor agents [22-24].

(Fig. 2. should be listed here)

Thus, in our continuing study, we synthesized a series of novel benzothiazole derivatives bearing the indole-based moiety with semicarbazone as a linker based on a structure-based molecular hybridization strategy, a commonly and effective approach to develop small molecules with multi-targeted molecular mechanism (Fig.3). Furthermore, some polar and water-soluble secondary amines were introduced at the 6-position of benzothiazole to avoid the metabolic pathway [19] and regulated the lipid-water partition coefficient of target compounds.

(Fig. 3. should be listed here)

Herein, thirty one novel benzothiazole derivatives bearing indole-based moiety and their antitumor activities in vitro against four cancer cell lines (HT-29, H460, A549 and MDA-MB-231) were reported. Additionally, the in vitro enzymatic assay and cell cycle analysis were also performed to investigate mechanism of action preliminarily. Furthermore, 3D-QSAR model was built to provide a comprehensive guide for further structural modification and optimization.

\section{Chemistry}

The preparation of key intermediates 14a-c is illustrated in Scheme 1. The commercially available material 4-nitrobenzyl bromide fused with excessive secondary amines (4-methyl piperidine, dimethylamine and diethylamine) in acetonitrile at room temperature for 3 hours to provide intermediates $\mathbf{1 0 a}-\mathbf{c}$, which were then reduced in the presence of $80 \%$ hydrazine, $\mathrm{FeCl}_{3} \cdot 6 \mathrm{H}_{2} \mathrm{O}$ to obtain the substituted anilines 11a-c. Compounds 11a-c were further cyclized with ammonium thiocyanate and bromine to give 12a-c in a satisfactory yield(65-88\%) according to the reported method[25]. Treatment of 12a-c with phenyl chloroformate though $\mathrm{N}$-acylation reaction yielded the 13a-c. Subsequently, the key intermediate semicarbazides 14a-c were generated via hydrazinolysis of 13a-c with $80 \%$ hydrazine monohydrate in 1,4-dioxane at $80{ }^{\circ} \mathrm{C}$ for 6 hours.

(Scheme 1. should be listed here)

As shown in Scheme 2, the $N$-substituted benzyl-1H-indole-3-carbaldehydes 16a-h were synthesized through nucleophilic substitution of various benzyl chloride with $1 \mathrm{H}$-indole-3-carboxaldehyde 15 in the presence of $\mathrm{K}_{2} \mathrm{CO}_{3}$ in $\mathrm{N}, \mathrm{N}$-dimethylformamide (DMF). The 5-substituted isatins 17a-c were prepared via condensation of aniline with chloral and further cyclization in sulfuric acid by referring literature procedure[26]. Finally, the target compounds 
18a-c, 19a-e and 20a-w were generated via the condensation of 14a-c with indole-3-carbaldehyde derivatives (15 and 16a-h) or 5-substituted isatins 17a-c in ethanol at $78{ }^{\circ} \mathrm{C}$ in the presence of catalytic amount of acetic acid[27].

(Scheme 2. should be listed here)

The chemical structures of the target compounds were confirmed by ${ }^{1} \mathrm{H}$ NMR, ${ }^{13} \mathrm{C}$ NMR, NOESY NMR, and MS spectra. All target compounds 18a-c and 20a-w could exist in either the $E$ or $Z$ isomeric form due to the imino bond, compound 20a was further selected to identify the stereochemistry by undergoing NOESY NMR. Results (Fig. 4 and supplementary information) showed that an evident NOE signal was observed between the $\mathrm{H}_{1}(-\mathrm{CH}=\mathrm{N}-, \delta=8.27 \mathrm{ppm})$ and $\mathrm{H}_{2}$ (=N-NH-, $\delta=11.13 \mathrm{ppm})$ in the $E$ isomer $(E-20 a)$, which should not be observed in the putative $\mathrm{Z}$ isomer due to the larger intramolecular H-H distance (Z-20a). Thus, target compounds 18a-c and 20a-w were unequivocally confirmed as the $E$ isomer.

(Fig. 4. should be listed here)

\section{Results and discussion}

\subsection{In vitro cytotoxicity evaluation}

All target compounds were evaluated for their in vitro antitumor activity against HT29 (human colon cancer cells), H460 (human lung cancer), A549 (non-small cell lung cancer) and MDA-MB-231 (human breast cancer) adopting MTT assay and using oncrasin-1 and PAC-1 as positive controls. The compounds were tested in three independent experiments and the results are presented as $\mathrm{IC}_{50}$ values (Table 1 ).

As illustrated in Table 1, the pharmacological results indicated that all the synthesized target compounds showed moderate to excellent cytotoxic activity against all tested cancer cell lines. It was observed that compounds 20a-w with benzyl- $1 H$-indole moiety displayed better activity compared to compounds 18a-c and 19a-e, as suggesting that the presence of $N$-benzyl group could dramatically enhance their antitumor activities. It was worth mentioning that nearly all the compounds with benzyl- $1 H$-indole moiety possessed significantly better selectivity against HT29 cancer cell line than other three cancer cell lines, indicating the good potency of these structures for the treatment of colon cancer. The most promising compound 20d exhibited the highest antitumor activity against HT29, H460, A549 and MDA-MB-231 with $\mathrm{IC}_{50}$ values of 0.024, 0.29, 0.84 and $0.88 \mu \mathrm{M}$, respectively.

Further investigations were performed to study the effect of different amine moieties of 20a-w against HT29 and H460 cancer cell lines on cytotoxic activity. As a general trend, it was observed that introduction of different secondary amino groups at the C-6 position of benzothiazole moiety had a notable influence on activity, with following rank order of potency: dimethylamine> diethylamine >4-methyl piperidine, which indicated that bulk of amine moieties $\left(\mathrm{R}_{1}\right)$ was critical for the activity.

Furthermore, electronic and steric effects of the $\mathrm{N}$-benzyl group were discussed via introduction of various $\mathrm{R}$ groups. The results revealed that increasing the size of substituent at the 4-position of benzyl group decreased the antitumor activity clearly $(20 \mathrm{~b} v s .20 \mathrm{c}, 20 \mathbf{j} v s .20 \mathrm{k}, 20 \mathrm{r}$ vs.20s), which suggested that steric hindrance of the group at this region of benzyl group exhibited a negative effect on the cytotoxic activity. Moreover, introduction of one-electron-withdrawing group (20d, 201, 20t, $\mathrm{R}=4-\mathrm{Cl}$ ) or weak mono-electron-donating group (20b, 20j, 20r, $\left.\mathrm{R}=4-\mathrm{CH}_{3}\right)$ had no significant change in antitumor activity, whereas incorporation of two-EWGs such as 
2,4-di-Cl or 3,4-di-Cl $(\mathbf{2 0 g}, \mathbf{2 0 h}, \mathbf{2 0 0}, \mathbf{2 0 p}, \mathbf{2 0 w})$ on the benzyl group resulted in a dramatic decrease in activity, implying that reducing the electron density of benzyl group was unfavorable for the activity. In addition, the activities of compounds 20d-f, 20m-o and 20v-x showed that one-EWG (-Cl) to the 3 or 4-position of benzyl group did a better contribution to the potency against HT29 and H460 than the 2-position.

In view of above observation, these encouraging results provided a valuable leading compound 20d with excellent antitumor activity, and highlight the potential for further development of novel benzothiazole derivatives bearing benzyl- $1 H$-indole moiety as potent antitumor agents.

(Table 1. should be listed here)

\subsection{In vitro enzymatic assays}

In order to investigate the molecular mechanism of action preliminarily, compounds 19a, 20d and 20e with potent activity were screened against 8 selected upstream and downstream protein kinases. The results summarized in Table 2 were expressed as percentage inhibition and were derived from two independent experiments.

As shown, compound 20d activated downstream procaspase-3 kinase by $70.9 \%$ at a concentration of $10 \mu \mathrm{M}$, whereas the impact on the other tested kinases was negligible. These results indicated that compound 20d might inhibit the growth of tumor cells via activation of procaspase-3. But the enzymatic potency of 20d can not justify for its strong and broad spectrum antitumor activity, as suggested the presence of other underlying mechanism that controlled the activity of this new compound against cancer cells.

(Table 2. should be listed here)

\subsection{Effects of $\mathbf{2 0 d}$ on cell cycle arrest in HT29 cells}

Intervention with deregulated cell cycle progression in cancer cells correlates well with the inhibition of cell proliferation, and targeting the cell cycle has been a growing area as a new approach for cancer therapy. Thus, cell cycle analysis was further performed to investigate the downstream molecular mechanism of compound 20d. Change of the cell cycle profile induced by 20d were studied using high content analysis system (HCA) with PI staining [28]. Cells were harvested on 48 hours after compound treatment at $10 \mu \mathrm{M}$ and analyzed for the distribution of G0/G1, S and G2/M phases of the cell cycle. Normally, healthy well-differentiated HT29 cell line would be $54.11 \%$ in G0/G1 phase, $9.35 \%$ in S phase, and $36.55 \%$ in G2/M phase (supplementary information). The results (Fig.5 and supplementary information) revealed that compound 20d showed apparent cell cycle arrest in G0/G1 phase (68.66\%) in HT29 cell lines, which implied that antitumor effect of $\mathbf{2 0 d}$ was associated with cell cycle arrest as a potential mechanism.

(Fig. 5. should be listed here)

In conclusion, enzymatic assay as well as cell cycle analysis indicated that the marked pharmacological activity of compound 20d might be ascribed to both activation of procaspase-3 (apoptosis-inducing) and cell cycle arrest. Although the upstream precise mechanism needed to be further investigated, the excellent antitumor activity of compound 20d recommended it as a lead compound which deserved further research with regard to its application in the treatment of human colon cancers.

\subsection{D-QSAR study}

In order to provide a reliable theoretical basis for the rational design of this novel series of compounds in our further structural modification and optimization, 3D-QSAR model was built 
and discussed by using Discovery Studio 3.0.

\subsubsection{D-QSAR model}

Molecular alignment was considered as a crucial step for 3D-QSAR study, so the optimal compound 20d was selected as template to build alignment conformation before building the QSAR model, the molecular alignment was shown in Fig. 6B, as shown, all the structures of target compounds were well aligned onto the common substructure 4-(benzothiazol-2-yl)semicarbazide nucleus (Fig. 6A).

(Fig. 6. should be listed here)

Based on the preliminary biological results, a 3D-QSAR model of target compounds was developed by using a novel 3D-QSAR protocol of Discovery Studio 3.0. Thirty one compounds (Table 1) with $\mathrm{IC}_{50}$ values ranging from 0.024 to $15.2 \mu \mathrm{M}$ for inhibiting HT29 were selected as the model dataset. Among them, twenty four compounds were selected as training set, seven compounds were selected as test set. The $\mathrm{IC}_{50}$ values were converted into corresponding $\mathrm{pIC}_{50}$ values as shown in supplementary information by the formula in Eq.: $\mathrm{pIC}_{50}=-\log _{10} \mathrm{IC}_{50}$.

The correlation coefficient $\mathrm{q}^{2}$ and $\mathrm{r}^{2}$ between observed and predicted activity of training set were found to be 0.850 and 0.987 , while $\mathrm{r}^{2}$ of test set was found to be 0.811 , which proved this QSAR model was acceptable. Predicted $\mathrm{pIC}_{50}$ values and residual errors of thirty one compounds by this QSAR model had been given in supplementary information. The plot of the predicted data versus the observed $\mathrm{pIC}_{50}$ of training set and test set was shown in Fig.7.

(Fig. 7. should be listed here)

\subsubsection{D-QSAR analysis}

The molecules aligned with the iso-surfaces of the 3D-QSAR model coefficients on van der Waals grids (Fig. 8A) and electrostatic potential grids (Fig. 8B) were listed. According to Fig.8, steric map (Fig. 8A) showed green contours under the $N$-position of indole ring, implying that bulky groups were favored at this position, validating that compounds 20a-w with $N$-benzyl group exhibited higher cytotoxic activity than compounds 18a-c. In addition, yellow contours around $\mathrm{R}^{1}$ group and at the 4-position substituent of benzyl group indicating bulky groups were not tolerated for these regions, which might explain that these compounds $\left(\mathrm{R}^{1}=4\right.$-methyl piperidine, $\mathrm{R}=$ 4-tert-butyl) were less potent. Furthermore, a yellow contour around the 5-position of indole moiety suggested that introduction of bulky groups was unhelpful for increasing the activity.

(Fig. 8. should be listed here)

Electrostatic map (Fig. 8B) was shown in blue around 2-position of benzyl group indicating that electronegative atom was disfavored at this positions, compounds $20 \mathrm{f}, 20 \mathrm{n}$ and $20 \mathrm{v}$ were good examples, but the red contour around $\mathrm{R}^{1}$ group and the 4-position of benzyl group suggested that negative charge group might play a favorable role on activity, combined with the steric map, it was clear that introduction of substituents with both small atom radius and negative charge at these regions would be beneficial for improving the antitumor activity.

\section{Conclusions}

In this paper, we designed and synthesized thirty one novel benzothiazole derivatives bearing indole-based moiety, and their in vitro antitumor activity was evaluated against four cancer cell lines (HT29, H460, A549, and MDA-MB-231). The results revealed that most compounds showed moderate to excellent activity against all tested cancer cell lines. Biological evaluation of the title compounds led to identification of compound 20d, which exhibited excellent antitumor activity, 
and might inhibit the growth of tumor cells via double mechanisms as both activation of procaspase-3 (apoptosis-inducing) and cell cycle arrest, and 20d had emerged as a valuable lead for further structural modifications. The SAR analyses indicated that introduction of $N$-benzyl group on the indole moiety could dramatically enhance the antitumor activity, and compounds with $N$-benzyl-indole moiety showed better selectivity against HT29 cancer cell line. In addition, steric effect of both amine moieties $\left(\mathrm{R}^{1}\right)$ and the substituent at 4-position of the benzyl group were responsible for the cytotoxic activity, and reducing the electron density of benzyl group was unfavorable for the antitumor activity. It was also confirmed that introduction of one-EWG at the 2-position of benzyl group was not tolerated. Furthermore, 3D-QSAR model (training set: $\mathrm{q}^{2}=0.850 \mathrm{r}^{2}=0.987$, test set: $\mathrm{r}^{2}=0.811$ ) was built to provide significant insight for guiding further structural modification and optimization. Studies on the mechanism of action of these compounds are in progress and will be reported in future.

\section{Experimental}

\subsection{Chemistry}

Unless otherwise specified, all melting points were obtained on a Büchi Melting Point B-540 apparatus (Büchi Labortechnik, Flawil, Switzerland) and were uncorrected. Mass spectra (MS) were taken in ESI mode on Agilent 1100 LC-MS (Agilent, Palo Alto, CA, USA.). ${ }^{1} \mathrm{H}$ NMR and ${ }^{13} \mathrm{C}$ NMR spectra were recorded on Bruker ARX-400, 400MHz or Bruker ARX-600, 600MHz spectrometers (Bruker Bioscience, Billerica, MA, USA) with TMS as an internal standard. Column chromatography was run on silica gel (200-300 mesh) from Qingdao Ocean Chemicals (Qingdao, Shandong, China). The IR spectra were recorded by means of the $\mathrm{KBr}$ pellet technique on a Bruker FTS 135 spectrometer. The elemental analysis of the compounds was performed on a Perkin Elmer 2400 Elemental Analyser. All materials were obtained from commercially available sources and were used without further purification.

\subsubsection{General procedure for preparation of compounds 10a-c}

4-Nitrobenzyl bromide $(10.8 \mathrm{~g}, 0.05 \mathrm{~mol})$ and excessive secondary amine $(50 \mathrm{~mL})$ were added in acetonitrile $(50 \mathrm{~mL})$. The reaction was stirred at room temperature for 3 hours, water $(100 \mathrm{~mL})$ was added, and extracted two times with dichloromethane (DCM). The organic extracts were combined, dried over sodium sulfate, filtered, and concentrated to obtain compounds 10a-c.

\subsubsection{N,N-dimethyl-1-(4-nitrophenyl)methanamine (10a)}

Yellow oil; Yield: 85\%; b.p.:146-148 ${ }^{\circ} \mathrm{C}$; MS (ESI) m/z: $181.1[\mathrm{M}+\mathrm{H}]^{+}$; ${ }^{1} \mathrm{H}$ NMR $(400 \mathrm{MHz}$, $\left.\mathrm{CDCl}_{3}\right) \delta 8.21-8.15\left(\mathrm{~m}, 2 \mathrm{H}, 2\right.$-phenyl-H), 7.52-7.45 (m, 2H, 2-phenyl-H), 3.55 (s, 2H, $-\mathrm{CH}_{2}$ ) , $2.28\left(\mathrm{~s}, 6 \mathrm{H}, 2-\mathrm{CH}_{3}\right)$.

\subsubsection{N-ethyl-N-(4-nitrobenzyl)ethanamine (10b)}

Yellow oil; Yield: 82\%; b.p.: 290-292 ${ }^{\circ} \mathrm{C}$; MS (ESI) m/z: $209.1[\mathrm{M}+\mathrm{H}]^{+} ;{ }^{1} \mathrm{H}$ NMR (400 MHz, $\left.\mathrm{CDCl}_{3}\right) \delta$ 8.21-8.13 (m, 2H, 2-phenyl-H), 7.56-7.49 (m, 2H, 2-phenyl-H), 3.69 (s, 2H, - $\mathrm{CH}_{2}$ ), 2.57-2.63 (q, $J=7.2 \mathrm{~Hz}, 4 \mathrm{H}, 2-\mathrm{CH}_{2^{-}}$), $1.07\left(\mathrm{t}, J=7.2 \mathrm{~Hz}, 6 \mathrm{H}, 2-\mathrm{CH}_{3}\right.$ ).

\subsubsection{4-Methyl-1-(4-nitrobenzyl)piperidine (10c)}

Yellow oil; Yield: $92 \%$; b.p.: 330-332 ${ }^{\circ} \mathrm{C}$; MS (ESI) m/z: $235.5[\mathrm{M}+\mathrm{H}]^{+} ;{ }^{1} \mathrm{H}$ NMR $(400 \mathrm{MHz}$, $\left.\mathrm{CDCl}_{3}\right) \delta$ 8.22-8.15 (m, 2H, 2-phenyl-H), 7.77-7.69 (m, 2H, 2-phenyl-H), 3.92 (s, 2H, - $\mathrm{CH}_{2}$ ),

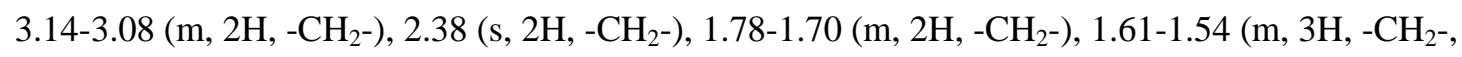
$-\mathrm{CH}-), 0.98\left(\mathrm{~d}, J=6.2 \mathrm{~Hz}, 3 \mathrm{H},-\mathrm{CH}_{3}\right)$.

\subsubsection{General procedure for preparation of compounds 11a-c}


A mixture of compounds 10a-c $(0.05 \mathrm{~mol})$ in ethanol $(100 \mathrm{~mL})$ was heated at $65{ }^{\circ} \mathrm{C}$, $\mathrm{FeCl}_{3} .6 \mathrm{H}_{2} \mathrm{O}(2.8 \mathrm{~g}, 0.001 \mathrm{~mol})$ and activated carbon $(0.18 \mathrm{~g}, 0.015 \mathrm{~mol})$ were added, and $80 \%$ hydrazine hydrate $(25 \mathrm{~g}, 0.5 \mathrm{~mol})$ was added drop wise at such a rate to keep the temperature below $70{ }^{\circ} \mathrm{C}$. The reaction was heated at reflux for 5 hours and then cooled to room temperature and concentrated. Water $(100 \mathrm{~mL})$ was added, the reaction solution was extracted three times with DCM. The organic extracts were combined, dried over sodium sulfate, filtered, and concentrated to obtain compounds 11a-c.

\subsubsection{4-((Dimethylamino)methyl)aniline (11a)}

Colorless oil; Yield: 78\%; b.p.: 221-223 ${ }^{\circ} \mathrm{C}$; MS (ESI) m/z: $150.6[\mathrm{M}+\mathrm{H}]^{+} ;{ }^{1} \mathrm{H}$ NMR (400 $\left.\mathrm{MHz}, \mathrm{CDCl}_{3}\right) \delta$ 7.09-7.04 (m, 2H, 2-phenyl-H), 6.66-6.60 (m, 2H, 2-phenyl-H), 3.63 (s, 2H, $\left.-\mathrm{CH}_{2}-\right), 3.32\left(\mathrm{~s}, 2 \mathrm{H},-\mathrm{NH}_{2}\right), 2.21\left(\mathrm{~s}, 6 \mathrm{H},-\mathrm{CH}_{3}\right)$.

\subsubsection{4-((Diethylamino)methyl)aniline (11b)}

Colorless oil; Yield: 74\%; m.p.:46-47 ${ }^{\circ} \mathrm{C}$; MS (ESI) m/z: $178.6[\mathrm{M}+\mathrm{H}]^{+} ;{ }^{1} \mathrm{H}$ NMR $(400 \mathrm{MHz}$, $\left.\mathrm{CDCl}_{3}\right) \delta$ 7.16-7.10 (m, 2H, 2-phenyl-H), 6.68-6.62 (m, 2H, 2-phenyl-H), 3.63 (s, 2H, - $\mathrm{CH}_{2}-$ ), $3.50\left(\mathrm{~s}, 2 \mathrm{H},-\mathrm{NH}_{2}\right), 2.56-2.50$ (q, $\left.J=7.2 \mathrm{~Hz}, 4 \mathrm{H}, 2-\mathrm{CH}_{2}-\right), 1.06\left(\mathrm{t}, J=7.2 \mathrm{~Hz}, 6 \mathrm{H},-\mathrm{CH}_{3}\right)$.

\subsubsection{4-((4-Methylpiperidin-1-yl)methyl)aniline (11c)}

Colorless oil; Yield: 83\%; b.p.: 303-305 ${ }^{\circ} \mathrm{C}$; MS (ESI) m/z: $204.5[\mathrm{M}+\mathrm{H}]^{+} ;{ }^{1} \mathrm{H}$ NMR (400 $\left.\mathrm{MHz}, \mathrm{CDCl}_{3}\right) \delta$ 7.15-7.09 (m, 2H, 2-phenyl-H), 6.66-6.60 (m, 2H, 2-phenyl-H), 3.60 (s, 2H, $\left.-\mathrm{CH}_{2^{-}}\right), 3.38\left(\mathrm{~s}, 2 \mathrm{H},-\mathrm{NH}_{2}\right), 2.89-2.80\left(\mathrm{~m}, 2 \mathrm{H},-\mathrm{CH}_{2^{-}}\right), 1.95-1.87\left(\mathrm{~m}, 2 \mathrm{H},-\mathrm{CH}_{2^{-}}\right), 1.58-1.49(\mathrm{~m}, 2 \mathrm{H}$, $\left.-\mathrm{CH}_{2}-\right), 1.33-1.32(\mathrm{~m}, 1 \mathrm{H},-\mathrm{CH}-), 1.29-1.19\left(\mathrm{~m}, 2 \mathrm{H},-\mathrm{CH}_{2}-\right), 0.90\left(\mathrm{~d}, J=6.2 \mathrm{~Hz}, 3 \mathrm{H},-\mathrm{CH}_{3}\right)$.

\subsubsection{General procedure for preparation of compounds 12a-c}

A mixture of compounds 11a-c $(0.05 \mathrm{~mol})$ and $\mathrm{NH}_{4} \mathrm{SCN}(19.03 \mathrm{~g}, 0.25 \mathrm{~mol})$ in glacial acetic acid $(100 \mathrm{~mL})$ was cooled to $10{ }^{\circ} \mathrm{C}$ in an ice bath and stirred for 10-20 minute. Then bromine $(2.82 \mathrm{~mL}, 0.055 \mathrm{~mol})$ in glacial acetic acid was added drop wise at such a rate to keep the temperature below $10^{\circ} \mathrm{C}$. The mixture was stirred at room temperature for 4-6 hours and then poured into hot water $(500 \mathrm{~mL})$, and basified to $\mathrm{pH} 11.0$ with ammonia solution $\left(\mathrm{NH}_{4} \mathrm{OH}\right)$. The resulting precipitate was filtered, washed with water and dried to get a light yellow to brown solid. The crude product was purified by chromatography on silica gel using $\mathrm{MeOH} / \mathrm{CH}_{2} \mathrm{Cl}_{2}$ to afford compounds 12a-c.

\subsubsection{6-((Dimethylamino)methyl)benzothiazol-2-amine (12a)}

Light yellow solid; Yield: 65\%; m.p.:141-143 ${ }^{\circ} \mathrm{C}$; MS (ESI) m/z: $208.03[\mathrm{M}+\mathrm{H}]^{+} ;{ }^{1} \mathrm{H}$ NMR $\left(400 \mathrm{MHz}, \mathrm{DMSO}-d_{6}\right) \delta 9.51\left(\mathrm{~s}, 2 \mathrm{H},-\mathrm{NH}_{2}\right), 7.99(\mathrm{~d}, J=1.2 \mathrm{~Hz}, 1 \mathrm{H}$, benzothiazole-H), 7.61 (dd, $J$ $=8.4,1.2 \mathrm{~Hz}, 1 \mathrm{H}$, benzothiazole- $\mathrm{H}), 7.55(\mathrm{~d}, J=8.4 \mathrm{~Hz}, 1 \mathrm{H}$, benzothiazole- $\mathrm{H}), 4.33(\mathrm{~s}, 2 \mathrm{H}$, $\left.-\mathrm{CH}_{2}-\right), 2.70\left(\mathrm{~s}, 3 \mathrm{H},-\mathrm{CH}_{3}\right), 2.69\left(\mathrm{~s}, 3 \mathrm{H},-\mathrm{CH}_{3}\right)$.

\subsubsection{6-((Diethylamino)methyl)benzothiazol-2-amine (12b)}

Light yellow solid; Yield: 88\%; m.p.:136-138 ${ }^{\circ} \mathrm{C}$; MS (ESI) m/z: $236.07[\mathrm{M}+\mathrm{H}]^{+} ;{ }^{1} \mathrm{H}$ NMR $\left(400 \mathrm{MHz}, \mathrm{DMSO}-d_{6}\right) \delta 7.55\left(\mathrm{~s}, 1 \mathrm{H}\right.$, benzothiazole-H), $7.37\left(\mathrm{~s}, 2 \mathrm{H},-\mathrm{NH}_{2}\right), 7.26(\mathrm{~d}, J=8.2 \mathrm{~Hz}, 1 \mathrm{H}$, benzothiazole-H), 7.14 (dd, $J=8.2,1.4 \mathrm{~Hz}, 1 \mathrm{H}$, benzothiazole-H), $3.52\left(\mathrm{~s}, 2 \mathrm{H},-\mathrm{CH}_{2}-\right), 2.47-2.42$ (q, $\left.J=7.2 \mathrm{~Hz}, 4 \mathrm{H}, 2-\mathrm{CH}_{2}-\right), 0.97\left(\mathrm{t}, J=7.2 \mathrm{~Hz}, 6 \mathrm{H}, 2-\mathrm{CH}_{3}\right)$.

\subsubsection{6-((4-Methylpiperidin-1-yl)methyl)benzothiazol-2-amine (12c)}

Yellow solid; Yield: 85\%; m.p.:201-203 ${ }^{\circ} \mathrm{C}$; MS (ESI) m/z: $262.13[\mathrm{M}+\mathrm{H}]^{+} ;{ }^{1} \mathrm{H}$ NMR $(400$ MHz, DMSO- $\left.d_{6}\right) \delta 7.75\left(\mathrm{~s}, 1 \mathrm{H}\right.$, benzothiazole-H), $7.65\left(\mathrm{~s}, 2 \mathrm{H},-\mathrm{NH}_{2}\right), 7.38(\mathrm{~d}, J=8.2 \mathrm{~Hz}, 1 \mathrm{H}$, benzothiazole- $\mathrm{H}), 7.30(\mathrm{~d}, J=8.2 \mathrm{~Hz}, 1 \mathrm{H}$, benzothiazole- $\mathrm{H}), 4.22\left(\mathrm{~s}, 2 \mathrm{H},-\mathrm{CH}_{2}-\right), 3.33(\mathrm{~s}, 2 \mathrm{H}$, $\left.-\mathrm{CH}_{2^{-}}\right), 2.77\left(\mathrm{~s}, 2 \mathrm{H},-\mathrm{CH}_{2^{-}}\right), 1.81-1.74\left(\mathrm{~m}, 2 \mathrm{H},-\mathrm{CH}_{2^{-}}\right), 1.56(\mathrm{~s}, 1 \mathrm{H},-\mathrm{CH}-), 1.31-1.25(\mathrm{~m}, 2 \mathrm{H}$, 
$\left.-\mathrm{CH}_{2}-\right), 0.90\left(\mathrm{~d}, J=5.6 \mathrm{~Hz}, 3 \mathrm{H},-\mathrm{CH}_{3}\right)$.

\subsubsection{General procedure for preparation of compounds 13a-c}

Compounds 12a-c $(0.01 \mathrm{~mol})$ and pyridine $(0.8 \mathrm{~mL}, 0.02 \mathrm{~mol})$ in DCM $(20 \mathrm{~mL})$ were cooled to $0{ }^{\circ} \mathrm{C}$ in an ice bath. The mixture was stirred for 0.5 hour, phenyl chloroformate $(0.015 \mathrm{~mol})$ was added drop wise at such a rate to keep the temperature below $10{ }^{\circ} \mathrm{C}$. The reaction was stirred at room temperature for 4-6 hours and filtered. The white to light yellow solid was collected and washed with DCM to obtain compounds 13a-c.

\subsubsection{Phenyl-6-((dimethylamino)methyl)benzothiazol-2-ylcarbamate (13a)}

White solid; Yield: 94\%; m.p.:178-180 ${ }^{\circ} \mathrm{C}$; MS (ESI) m/z: $328.29[\mathrm{M}+\mathrm{H}]^{+}$; ${ }^{1} \mathrm{H}$ NMR (400 MHz, DMSO- $\left.d_{6}\right) \delta 10.60(\mathrm{~s}, 1 \mathrm{H},-\mathrm{NH}-), 8.15(\mathrm{~s}, 1 \mathrm{H}$, benzothiazole-H), $7.82(\mathrm{~d}, J=8.4 \mathrm{~Hz}, 1 \mathrm{H}$, benzothiazole-H), $7.64(\mathrm{~d}, J=8.4 \mathrm{~Hz}, 1 \mathrm{H}$, benzothiazole- $\mathrm{H}), 7.51-7.45(\mathrm{~m}, 2 \mathrm{H}, 2-$ phenyl-H), 7.36-7.30 (m, 3H, 3-phenyl-H), 4.37 (s, $\left.2 \mathrm{H},-\mathrm{CH}_{2}-\right), 2.71\left(\mathrm{~s}, 3 \mathrm{H},-\mathrm{CH}_{3}\right), 2.70\left(\mathrm{~s}, 3 \mathrm{H},-\mathrm{CH}_{3}\right)$.

\subsubsection{Phenyl-6-((diethylamino)methyl)benzothiazol-2-ylcarbamate (13b)}

White solid; Yield: 92\%; m.p.:207-209 ${ }^{\circ} \mathrm{C}$; MS (ESI) m/z: 356.16[M+H $]^{+}$; ${ }^{1} \mathrm{H}$ NMR (400 MHz, DMSO- $\left.d_{6}\right) \delta 10.41(\mathrm{~s}, 1 \mathrm{H},-\mathrm{NH}-), 8.22(\mathrm{~d}, J=1.6 \mathrm{~Hz}, 1 \mathrm{H}$, benzothiazole-H), $7.82(\mathrm{~d}, J=$ $8.4 \mathrm{~Hz}, 1 \mathrm{H}$, benzothiazole- $\mathrm{H}), 7.70(\mathrm{dd}, J=8.4,1.6 \mathrm{~Hz}, 1 \mathrm{H}$, benzothiazole- $\mathrm{H}), 7.50-7.45(\mathrm{~m}, 2 \mathrm{H}$, 2-phenyl-H), 7.37-7.29 (m, 3H, 3-phenyl-H), 4.38 (s, 2H, - $\mathrm{CH}_{2}$ ), 3.10-3.04 (m, 4H, 2- $\left.\mathrm{CH}_{2}{ }^{-}\right), 1.27$ (t, $J=7.2 \mathrm{~Hz}, 6 \mathrm{H}, 2-\mathrm{CH}_{3}$ ).

\subsubsection{Phenyl-6-((4-methylpiperidin-1-yl)methyl)benzothiazol-2-ylcarbamate (13c)}

White solid; Yield: 97\%; m.p.:159-161 ${ }^{\circ} \mathrm{C}$; MS (ESI) m/z: 382.21[M+H] ${ }^{+} ;{ }^{1} \mathrm{H}$ NMR $(400$ MHz, DMSO- $\left.d_{6}\right) \delta 9.92(\mathrm{~s}, 1 \mathrm{H},-\mathrm{NH}-), 8.14(\mathrm{~s}, 1 \mathrm{H}$, benzothiazole- $\mathrm{H}), 7.82(\mathrm{~d}, J=8.4 \mathrm{~Hz}, 1 \mathrm{H}$, benzothiazole-H), $7.62(\mathrm{~d}, J=8.4 \mathrm{~Hz}, 1 \mathrm{H}$, benzothiazole- $\mathrm{H}), 7.50-7.44(\mathrm{~m}, 2 \mathrm{H}, 2-$ phenyl-H), 7.36-7.30 (m, 3H, 3-phenyl-H), 4.35 (s, $\left.2 \mathrm{H},-\mathrm{CH}_{2^{-}}\right), 3.36-3.30\left(\mathrm{~m}, 2 \mathrm{H},-\mathrm{CH}_{2}-\right), 2.94-2.88(\mathrm{~m}, 2 \mathrm{H}$, $\left.-\mathrm{CH}_{2^{-}}\right), 1.80-1.74\left(\mathrm{~m}, 2 \mathrm{H},-\mathrm{CH}_{2^{-}}\right), 1.59(\mathrm{~s}, 1 \mathrm{H},-\mathrm{CH}-), 1.42-1.36\left(\mathrm{~m}, 2 \mathrm{H},-\mathrm{CH}_{2^{-}}\right), 0.90(\mathrm{~d}, J=6.4$ $\left.\mathrm{Hz}, 3 \mathrm{H},-\mathrm{CH}_{3}\right)$.

\subsubsection{General procedure for preparation of compounds $14 a-c$}

A mixture of the compounds 13a-c $(0.01 \mathrm{~mol})$ and $80 \%$ hydrazine hydrate $(1.29 \mathrm{~mL}, 0.02$ mol) in 1,4-dioxane $(20 \mathrm{~mL})$ was heated at $80{ }^{\circ} \mathrm{C}$ for 6 hours, then the reaction mixture was cooled to room temperature and concentrated, diethyl ether $(20 \mathrm{~mL})$ was added and stirred for 0.5 hour and filtered, a white to gray solid was collected and washed with a small amount of water. The crude product was purified by chromatography on silica gel using $\mathrm{MeOH} / \mathrm{CH}_{2} \mathrm{Cl}_{2}$ to get compounds 14a-c.

5.1.5.1. 4-(6-((Dimethylamino)methyl)benzothiazol-2-yl)semicarbazide (14a)

White solid; Yield: $82 \%$; m.p.:183-185 ${ }^{\circ} \mathrm{C}$; MS (ESI) m/z: $265.7[\mathrm{M}+\mathrm{H}]^{+} ;{ }^{1} \mathrm{H}$ NMR $(400$ MHz, DMSO- $\left.d_{6}\right) \delta 8.37$ (s, $\left.1 \mathrm{H},-\mathrm{NH}-\right), 7.77$ (s, $1 \mathrm{H}$, benzothiazole-H), $7.56(\mathrm{~d}, J=8.2 \mathrm{~Hz}, 1 \mathrm{H}$, benzothiazole-H), $7.28(\mathrm{~d}, J=8.2 \mathrm{~Hz}, 1 \mathrm{H}$, benzothiazole- $\mathrm{H}), 3.44\left(\mathrm{~s}, 2 \mathrm{H},-\mathrm{CH}_{2^{-}}\right), 2.15(\mathrm{~s}, 6 \mathrm{H}$, 2- $\left.\mathrm{CH}_{3}\right)$.

\subsubsection{4-(6-((Diethylamino)methyl)benzothiazol-2-yl)semicarbazide (14b)}

White solid; Yield: $86 \%$; m.p.:128-130 ${ }^{\circ} \mathrm{C}$; MS (ESI) m/z: $607.9[2 \mathrm{M}+\mathrm{Na}]^{+} ;{ }^{1} \mathrm{H}$ NMR $(400$ MHz, DMSO- $\left.d_{6}\right) \delta 9.34$ (s, 1H, -NH-), 8.49 (s, 1H, -NH-), 8.03 (s, 1H, benzothiazole-H), 7.70 (d, $J=8.0 \mathrm{~Hz}, 1 \mathrm{H}$, benzothiazole-H), $7.50(\mathrm{~d}, J=8.0 \mathrm{~Hz}, 1 \mathrm{H}$, benzothiazole- $\mathrm{H}), 4.31\left(\mathrm{~s}, 2 \mathrm{H},-\mathrm{CH}_{2}-\right)$, $3.04\left(\mathrm{~s}, 4 \mathrm{H}, 2-\mathrm{CH}_{2}-\right), 1.22\left(\mathrm{~s}, 6 \mathrm{H}, 2-\mathrm{CH}_{3}\right)$.

\subsubsection{4-(6-((4-Methylpiperidin-1-yl)methyl)benzothiazol-2-yl)semicarbazide (14c)}

White solid; Yield: $76 \%$; m.p.:142-144 ${ }^{\circ} \mathrm{C}$; MS (ESI) m/z: $319.6[\mathrm{M}+\mathrm{H}]^{+} ;{ }^{1} \mathrm{H}$ NMR (400 
MHz, DMSO- $\left.d_{6}\right) \delta 9.33$ (s, 1H, -NH-), 7.79 (s, 1H, benzothiazole-H), 7.57 (d, $J=8.0 \mathrm{~Hz}, 1 \mathrm{H}$, benzothiazole-H), 7.31 (d, $J=7.2 \mathrm{~Hz}, 1 \mathrm{H}$, benzothiazole-H), 7.16 (t, $J=7.6 \mathrm{~Hz}, 1 \mathrm{H},-\mathrm{NH}-), 6.76$ (s, $\left.1 \mathrm{H},-\mathrm{NH}_{2}\right), 6.74\left(\mathrm{~s}, 1 \mathrm{H},-\mathrm{NH}_{2}\right), 3.54\left(\mathrm{~s}, 2 \mathrm{H},-\mathrm{CH}_{2^{-}}\right), 2.81\left(\mathrm{~s}, 2 \mathrm{H},-\mathrm{CH}_{2^{-}}\right), 1.94\left(\mathrm{~s}, 2 \mathrm{H},-\mathrm{CH}_{2^{-}}\right)$, 1.60-1.52 (m, 2H, - $\left.\mathrm{CH}_{2^{-}}\right), 1.35$ (s, $\left.1 \mathrm{H},-\mathrm{CH}-\right), 1.19-1.13\left(\mathrm{~m}, 2 \mathrm{H},-\mathrm{CH}_{2^{-}}\right), 0.88(\mathrm{~d}, J=6.4 \mathrm{~Hz}, 3 \mathrm{H}$, $\left.-\mathrm{CH}_{3}\right)$.

\subsubsection{General procedure for the preparation of compounds $\mathbf{1 6 a - h}$}

$1 H$-Indole-3-carbaldehyde 15 (14.5 g, $0.1 \mathrm{~mol})$, the appropriate benzyl chloride $(0.11 \mathrm{~mol})$ and anhydrous $\mathrm{K}_{2} \mathrm{CO}_{3}(27.6 \mathrm{~g}, 0.2 \mathrm{~mol})$ in $N, N$-dimethylformamide $(30 \mathrm{~mL})$ were heated at $80{ }^{\circ} \mathrm{C}$ for 5-8 hours. Upon cooling to room temperature, the mixture was poured into ice-water $(200 \mathrm{~mL})$ and then the precipitated was collected by filtration and dried. The crude product was purified by recrystallized from ethanol to give solids $\mathbf{1 6 a}-\mathbf{h}$.

\subsubsection{1-Benzyl-1H-indole-3-carbaldehyde (16a)}

White solid; Yield: 73\%; m.p.:106-108 ${ }^{\circ} \mathrm{C}$; MS (ESI) m/z: 234.2 [M-H] ${ }^{-}$

\subsubsection{1-(4-Methylbenzyl)-1H-indole-3-carbaldehyde (16b)}

White solid; Yield: 80\%; m.p.:105-107 ${ }^{\circ} \mathrm{C}$; MS (ESI) m/z: 248.3 [M-H] $]^{-}$

5.1.6.3. 1-(4-Tert-butylbenzyl)-1H-indole-3-carbaldehyde (16c)

White solid; Yield: 82\%; m.p.:112-114 ${ }^{\circ} \mathrm{C}$; MS (ESI) m/z: 290.4 [M-H] $]^{-}$ 5.1.6.4. 1-(4-Chlorobenzyl)-1H-indole-3-carbaldehyde (16d)

White solid; Yield: 79\%; m.p.:117-119 ${ }^{\circ} \mathrm{C}$; MS (ESI) m/z: 268.7 [M-H] $]^{-}$

\subsubsection{1-(3-Chlorobenzyl)-1H-indole-3-carbaldehyde (16e)}

White solid; Yield: 77\%; m.p.:78-80 C; MS (ESI) m/z: 268.8 [M-H] ${ }^{\circ}$.

\subsubsection{1-(2-Chlorobenzyl)-1H-indole-3-carbaldehyde (16f)}

White solid; Yield: 82\%; m.p.:131-133 ${ }^{\circ} \mathrm{C}$; MS (ESI) m/z: 268.7 [M-H] $]^{-}$

\subsubsection{1-(2,4-Dichlorobenzyl)-1H-indole-3-carbaldehyde (16g)}

White solid; Yield: 69\%; m.p.:136-138 ${ }^{\circ} \mathrm{C}$; MS (ESI) m/z: 303.2 [M-H] $]^{-}$ 5.1.6.8. 1-(3,4-Dichlorobenzyl)-1H-indole-3-carbaldehyde (16h)

White solid; Yield: 84\%; m.p.:133-135 ${ }^{\circ} \mathrm{C}$; MS (ESI) m/z: 303.1 [M-H] ${ }^{-}$.

\subsubsection{General procedure for the preparation of compounds 17a-c}

A mixture of substituted aniline $(0.05 \mathrm{~mol})$, hydroxylammonium chloride $(0.15 \mathrm{~mol}), \mathrm{Na}_{2} \mathrm{SO}_{4}$ ( $0.35 \mathrm{~mol}), 2 \mathrm{~mol} / \mathrm{L}$ hydrochloric acid $(5 \mathrm{~mL})$ and chloral hydrate $(0.06 \mathrm{~mol})$ were stirred in $\mathrm{H}_{2} \mathrm{O}$ $(250 \mathrm{~mL})$ at $95{ }^{\circ} \mathrm{C}$. The progress of the reaction was monitored by TLC (petroleum ether/ethyl acetate). After the reaction finished and cooled to room temperature. The reaction mixture was filtered and dried. The crude product was used directly for the next step without further purification.

To a flask $(100 \mathrm{~mL})$ which contained concentrated sulfuric acid $(20 \mathrm{~mL})$ was added $\mathrm{N}$-2-(hydroxyimino) acetamide derivative $(7.0 \mathrm{~g})$ in portions at $50{ }^{\circ} \mathrm{C}$ with vigorous stirring. The reaction temperature was maintained at $50-75{ }^{\circ} \mathrm{C}$ during the addition. After the addition was completed, the mixture was heated at $80{ }^{\circ} \mathrm{C}$ for 30 minute. The reaction mixture was cooled to room temperature and then poured into ice $(250 \mathrm{~g})$. The solid was filtered out and dried to yield the crude, which was purified by dissolving in dilute sodium hydroxide $(5 \%, 100 \mathrm{~mL})$ followed by acidified with $4 \mathrm{~mol} / \mathrm{L}$ hydrochloric acid $(20 \mathrm{~mL})$. The solid was filtered and dried to provide purified compounds $\mathbf{1 7 a - c .}$

\subsubsection{5-Fluoroindoline-2,3-dione (17a)}

Brown solid; Yield: 70\%; m.p.:224-226 ${ }^{\circ} \mathrm{C}$; MS (ESI) m/z: $164.1[\mathrm{M}-\mathrm{H}]^{-}$. 


\subsubsection{5-Chloroindoline-2,3-dione (17b)}

Yellow solid; Yield: 59\%; m.p.:255-257 C; MS (ESI) m/z: $180.1[\mathrm{M}-\mathrm{H}]^{-}$.

\subsubsection{5-(Trifluoromethoxy)indoline-2,3-dione (17c)}

Yellow solid; Yield: 68\%; m.p.:191-193 C; MS (ESI) m/z: $230.1[\mathrm{M}-\mathrm{H}]^{-}$.

5.1.8. General procedure for preparation of compounds 18a-c, 19a-e and 20a-w.

A mixture of compounds 14a-c $(0.001 \mathrm{~mol})$, the appropriate aromatic aldehyde 15 and 16a-h or 5 -substituted isatins $\mathbf{1 7 a - c}(0.0011 \mathrm{~mol})$ and one drop of glacial acetic acid in ethanol $(10 \mathrm{~mL})$ was heated at reflux for 6 hours and then cooled to room temperature and filtered. The obtained cake was washed with diethyl ether to get a white to light yellow solid. The crude product was purified by chromatography on silica gel using $\mathrm{MeOH} / \mathrm{CH}_{2} \mathrm{Cl}_{2}$ to afford compounds 18a-c, 19a-e and 20a-w.

5.1.8.1.(E)-4-(6-((dimethylamino)methyl)benzothiazol-2-yl)-1-((1H-indol-3-yl)methylene $)$ semicarb azide (18a)

Yield: 56\%; M.p.: 167-169 ${ }^{\circ} \mathrm{C}$; MS (ESI) m/z: $393.1[\mathrm{M}+\mathrm{H}]^{+} ;{ }^{1} \mathrm{H}$ NMR (400 MHz, DMSO- $\left.d_{6}\right)$ $\delta 11.64$ (s, 1H, -NH-), 11.07 (s, 1H, -NH-), 10.37 (s, 1H, indole-NH-), 8.27 (s, 1H, -CH=), 8.25 (d, $J=6.8 \mathrm{~Hz}, 1 \mathrm{H}$, indole-H), 7.95-7.83 (m, 2H, benzothiazole- $\mathrm{H}$, indole-H), $7.68(\mathrm{~d}, J=8.0 \mathrm{~Hz}, 1 \mathrm{H}$, benzothiazole-H), $7.46(\mathrm{~d}, J=6.8 \mathrm{~Hz}, 1 \mathrm{H}$, benzothiazole- $\mathrm{H}), 7.37(\mathrm{~d}, J=8.0 \mathrm{~Hz}, 1 \mathrm{H}$, indole-H), 7.28-7.14 (m, 2H, 2-indole-H), $3.63\left(\mathrm{~s}, 2 \mathrm{H},-\mathrm{CH}_{2}-\right), 2.27$ (s, 6H, 2- $\left.\mathrm{CH}_{3}\right)$; Anal. Calcd, for $\mathrm{C}_{20} \mathrm{H}_{20} \mathrm{~N}_{6} \mathrm{OS}(\%): \mathrm{C}, 61.20 ; \mathrm{H}, 5.14 ; \mathrm{N}, 21.41$. Found (\%):C, 61.10; H, 5.24; N, 21.33.

5.1.8.2.(E)-4-(6-((diethylamino)methyl)benzothiazol-2-yl)-1-((1H-indol-3-yl)methylene $)$ semicarba zide $(\mathbf{1 8 b})$

Yield: 58\%; M.p.: 159-161 ${ }^{\circ} \mathrm{C}$; MS (ESI) m/z: $421.1[\mathrm{M}+\mathrm{H}]^{+} ;{ }^{1} \mathrm{H}$ NMR $\left(400 \mathrm{MHz}\right.$, DMSO- $\left.d_{6}\right)$ $\delta 11.65(\mathrm{~s}, 1 \mathrm{H},-\mathrm{NH}-), 11.07$ (s, 1H, -NH-), 10.35 (s, 1H, indole-NH-), 8.27 (s, 1H, -CH=), 8.27-8.21 $(\mathrm{m}, 1 \mathrm{H}$, indole- $\mathrm{H}), 7.89(\mathrm{~s}, 2 \mathrm{H}$, benzothiazole- $\mathrm{H}$, indole- $\mathrm{H}), 7.68(\mathrm{~s}, 1 \mathrm{H}$, benzothiazole- $\mathrm{H})$, 7.48-7.44 $(\mathrm{m}, 1 \mathrm{H}$, benzothiazole- $\mathrm{H}), 7.42(\mathrm{~s}, 1 \mathrm{H}$, indole- $\mathrm{H}), 7.26-7.17(\mathrm{~m}, 2 \mathrm{H}$, 2-indole-H), 3.68 (s, $\left.2 \mathrm{H},-\mathrm{CH}_{2}-\right), 2.67\left(\mathrm{~s}, 4 \mathrm{H}, 2-\mathrm{CH}_{2}-\right), 1.06$ (s, 6H, 2- $\left.\mathrm{CH}_{3}\right)$; Anal. Calcd, for $\mathrm{C}_{22} \mathrm{H}_{24} \mathrm{~N}_{6} \mathrm{OS}(\%): \mathrm{C}, 62.83 ; \mathrm{H}, 5.75 ; \mathrm{N}, 19.98$. Found (\%):C, 62.89; H, 5.85; N, 19.90.

5.1.8.3.(E)-4-(6-((4-methylpiperidin-1-yl)methyl)benzothiazol-2-yl)-1-((1H-indol-3-yl)methylene)s emicarbazide $(\mathbf{1 8 c})$

Yield: 58\%; M.p.: 150-152 ${ }^{\circ} \mathrm{C}$; MS (ESI) m/z: $447.2[\mathrm{M}+\mathrm{H}]^{+} ;{ }^{1} \mathrm{H}$ NMR (400 MHz, DMSO- $\left.d_{6}\right)$ $\delta 11.64$ (s, 1H, -NH-), 11.14 (s, 1H, -NH-), 9.20 (s, 1H, indole-NH-), 8.28 (s, 1H, -CH=), 8.26 (d, $J=7.2 \mathrm{~Hz}, 1 \mathrm{H}$, indole-H), 8.07 (s, $1 \mathrm{H}$, indole-H), 7.89 (s, $1 \mathrm{H}$, benzothiazole-H), 7.79 (d, $J=7.8$ $\mathrm{Hz}, 1 \mathrm{H}$, benzothiazole- $\mathrm{H}), 7.53(\mathrm{~d}, J=7.8 \mathrm{~Hz}, 1 \mathrm{H}$, benzothiazole- $\mathrm{H}), 7.46(\mathrm{~d}, J=7.2 \mathrm{~Hz}, 1 \mathrm{H}$, indole- $\mathrm{H}$ ), 7.25-7.18 (m, 2H, 2-indole-H), $4.36\left(\mathrm{~s}, 2 \mathrm{H},-\mathrm{CH}_{2}-\right), 3.36$ (d, $J=10.8 \mathrm{~Hz}, 2 \mathrm{H},-\mathrm{CH}_{2}-$ ), 3.01-2.87 (m, 2H, - $\left.\mathrm{CH}_{2^{-}}\right), 1.79$ (d, $\left.J=10.8 \mathrm{~Hz}, 2 \mathrm{H},-\mathrm{CH}_{2^{-}}\right), 1.58(\mathrm{~s}, 1 \mathrm{H},-\mathrm{CH}-), 1.39-1.23(\mathrm{~m}, 2 \mathrm{H}$, $\left.-\mathrm{CH}_{2^{-}}\right), 0.90\left(\mathrm{~d}, J=6.4 \mathrm{~Hz}, 3 \mathrm{H},-\mathrm{CH}_{3}\right)$; Anal. Calcd, for $\mathrm{C}_{24} \mathrm{H}_{26} \mathrm{~N}_{6} \mathrm{OS}(\%): \mathrm{C}, 64.55 ; \mathrm{H}, 5.87 ; \mathrm{N}$, 18.82. Found (\%):C, 64.68; H, 5.94; N, 18.96.

5.1.8.4.4-(6-((Dimethylamino)methyl)benzothiazol-2-yl)-1-(5-fluoro-2-oxoindolin-3-ylidene)semic arbazide (19a)

Yield: 43\%; M.p.: 210-212 ${ }^{\circ} \mathrm{C}$; MS (ESI) m/z: 410.8 [M-H] ${ }^{-1}{ }^{1} \mathrm{H}$ NMR (600 MHz, DMSO- $\left.d_{6}\right)$ $\delta 12.62$ (s, 1H, -NH-), 11.29 (s, 1H, -NH-), 7.85 (s, 1H, benzothiazole-H), 7.59 (d, J= 8.2 Hz, 1H, benzothiazole- $\mathrm{H}), 7.47(\mathrm{~m}, 1 \mathrm{H}$, isatin- $\mathrm{H}), 7.36(\mathrm{~d}, J=8.2 \mathrm{~Hz}, 1 \mathrm{H}$, isatin- $\mathrm{H}), 7.17(\mathrm{~m}, 1 \mathrm{H}$, isatin- $\mathrm{H})$, $6.94(\mathrm{dd}, J=8.2,4.2 \mathrm{~Hz}, 1 \mathrm{H}$, benzothiazole- $\mathrm{H}), 3.64\left(\mathrm{~s}, 2 \mathrm{H},-\mathrm{CH}_{2}-\right), 2.26\left(\mathrm{~s}, 6 \mathrm{H}, 2-\mathrm{CH}_{3}\right)$; Anal. Calcd, for $\mathrm{C}_{19} \mathrm{H}_{17} \mathrm{FN}_{6} \mathrm{O}_{2} \mathrm{~S}(\%): \mathrm{C}, 55.33 ; \mathrm{H}, 4.15 ; \mathrm{N}, 20.38$. Found (\%):C, 55.43; H, 4.19; N, 20.29. 
5.1.8.5.4-(6-((Dimethylamino)methyl)benzothiazol-2-yl)-1-(5-chloro-2-oxoindolin-3-ylidene)semic arbazide $(\mathbf{1 9 b})$

Yield: $41 \%$; M.p.: $208-210{ }^{\circ} \mathrm{C}$; MS (ESI) m/z: 426.8 [M-H] ${ }^{-1}{ }^{1} \mathrm{H}$ NMR $\left(600 \mathrm{MHz}, \mathrm{DMSO}-d_{6}\right)$ $\delta 12.48(\mathrm{~s}, 1 \mathrm{H},-\mathrm{NH}-), 11.34(\mathrm{~s}, 1 \mathrm{H},-\mathrm{NH}-), 7.85(\mathrm{~s}, 1 \mathrm{H}$, benzothiazole-H), 7.74-7.68 (m, 1H, benzothiazole-H), $7.60(\mathrm{~d}, J=8.4 \mathrm{~Hz}, 1 \mathrm{H}$, isatin- $\mathrm{H}), 7.38(\mathrm{dd}, J=8.4,2.4 \mathrm{~Hz}, 1 \mathrm{H}$, isatin-H), 7.35 (d, $J=8.4 \mathrm{~Hz}, 1 \mathrm{H}$, isatin- $\mathrm{H}), 6.95(\mathrm{~d}, J=8.4 \mathrm{~Hz}, 1 \mathrm{H}$, benzothiazole- $\mathrm{H}), 3.59\left(\mathrm{~s}, 2 \mathrm{H},-\mathrm{CH}_{2^{-}}\right), 2.23$ (s, 6H, 2- $\mathrm{CH}_{3}$ ); Anal. Calcd, for $\mathrm{C}_{19} \mathrm{H}_{17} \mathrm{ClN}_{6} \mathrm{O}_{2} \mathrm{~S}(\%): \mathrm{C}, 53.21 ; \mathrm{H}, 4.00 ; \mathrm{N}, 19.59$. Found (\%):C, 53.29; H, 4.11; N, 19.48.

5.1.8.6.4-(6-((Diethylamino)methyl)benzothiazol-2-yl)-1-(5-fluoro-2-oxoindolin-3-ylidene)semicar bazide (19c)

Yield: 45\%; M.p.: 205-207 ${ }^{\circ} \mathrm{C}$; MS (ESI) m/z: 438.6 [M-H] $;{ }^{1}{ }^{H}$ NMR (400 MHz, DMSO- $d_{6}$ ) $\delta 12.53$ (s,1H, -NH-), 11.34 (s, 1H, -NH-), 7.90 (s, 1H, benzothiazole-H), 7.75 (d, J= 8.4 Hz 1H, benzothiazole-H), 7.53-7.50 (m, 2H, 2-isatin- $\mathrm{H}), 7.19(\mathrm{~m}, 1 \mathrm{H}$, isatin- $\mathrm{H}), 6.95$ (dd, $J=8.4,4.2 \mathrm{~Hz}$, $1 \mathrm{H}$, benzothiazole-H), $4.03\left(\mathrm{~s}, 2 \mathrm{H},-\mathrm{CH}_{2}-\right), 2.81\left(\mathrm{~d}, J=7.2 \mathrm{~Hz}, 4 \mathrm{H}, 2-\mathrm{CH}_{2}-\right), 1.14(\mathrm{t}, J=7.2 \mathrm{~Hz}, 6 \mathrm{H}$, 2- $\left.\mathrm{CH}_{3}\right)$; Anal. Calcd, for $\mathrm{C}_{21} \mathrm{H}_{21} \mathrm{FN}_{6} \mathrm{O}_{2} \mathrm{~S}(\%): \mathrm{C}, 57.26 ; \mathrm{H}, 4.81 ; \mathrm{N}, 19.08$. Found (\%):C, 57.36; H, $4.79 ; \mathrm{N}, 19.15$.

5.1.8.7.4-(6-((Diethylamino)methyl)benzothiazol-2-yl)-1-(5-(trifluoromethoxy)-2-oxoindolin-3-ylid ene)semicarbazide (19d)

Yield: 47\%; M.p.: 201-203 ${ }^{\circ} \mathrm{C}$; MS (ESI) m/z: 504.3 [M-H] ; ${ }^{1} \mathrm{H}$ NMR (400 MHz, DMSO- $d_{6}$ ) $\delta 12.49$ (s, 1H, -NH-), 11.40 (s, 1H, -NH-), 7.89 (s, 1H, benzothiazole-H), 7.79-7.51 (m, 2H, benzothiazole- $\mathrm{H}$, isatin- $\mathrm{H}), 7.41(\mathrm{~s}, 1 \mathrm{H}$, isatin- $\mathrm{H}), 7.34(\mathrm{~s}, 1 \mathrm{H}$, isatin- $\mathrm{H}), 7.02(\mathrm{~d}, J=6.4 \mathrm{~Hz}, 1 \mathrm{H}$, benzothiazole-H), $3.80\left(\mathrm{~s}, 2 \mathrm{H},-\mathrm{CH}_{2^{-}}\right), 2.62\left(\mathrm{~s}, 4 \mathrm{H}, 2-\mathrm{CH}_{2}-\right), 1.04$ (s, 6H, 2- $\left.\mathrm{CH}_{3}\right)$; Anal. Calcd, for $\mathrm{C}_{22} \mathrm{H}_{21} \mathrm{~F}_{3} \mathrm{~N}_{6} \mathrm{O}_{3} \mathrm{~S}(\%): \mathrm{C}, 52.17 ; \mathrm{H}, 4.18 ; \mathrm{N}, 16.59$. Found (\%):C, 52.25; H, 4.24; N, 16.49.

5.1.8.8.4-(6-((4-Methylpiperidin-1-yl)methyl)benzothiazol-2-yl)-1-(5-fluoro-2-oxoindolin-3-yliden e)semicarbazide (19e)

Yield: 47\%; M.p.: 190-192 ${ }^{\circ} \mathrm{C}$; MS (ESI) m/z: 464.7 [M-H] ; ${ }^{1} \mathrm{H}$ NMR $\left(600 \mathrm{MHz}, \mathrm{DMSO}-d_{6}\right)$ $\delta 12.59$ (s, 1H, -NH-), 11.27 (s, 1H, -NH-), 7.95 (s, 1H, benzothiazole-H), 7.66-7.61 (m, 1H, benzothiazole-H), 7.53-7.39 (m, 2H, 2-isatin-H), 7.18 (td, $J=9.6,3.0 \mathrm{~Hz}, 1 \mathrm{H}$, isatin-H), 6.93 (dd, $J=8.4,4.2 \mathrm{~Hz}, 1 \mathrm{H}$, benzothiazole- $\mathrm{H}), 3.88\left(\mathrm{~s}, 2 \mathrm{H},-\mathrm{CH}_{2^{-}}\right), 3.03-2.92\left(\mathrm{~m}, 2 \mathrm{H},-\mathrm{CH}_{2^{-}}\right), 2.44-2.27$

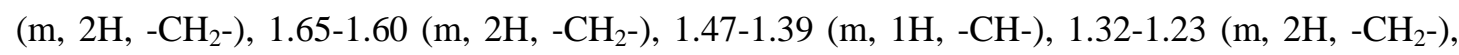
$0.87\left(\mathrm{~d}, J=6.6 \mathrm{~Hz}, 3 \mathrm{H},-\mathrm{CH}_{3}\right)$; Anal. Calcd, for $\mathrm{C}_{23} \mathrm{H}_{23} \mathrm{FN}_{6} \mathrm{O}_{2} \mathrm{~S}(\%): \mathrm{C}, 59.21 ; \mathrm{H}, 4.97 ; \mathrm{N}, 18.01$. Found (\%):C, 59.29; H, 4.87; N, 18.12 .

5.1.8.9.(E)-4-(6-((dimethylamino)methyl)benzothiazol-2-yl)-1-((1-benzyl-1H-indol-3-yl)methylene )semicarbazide (20a)

Yield: 52\%; M.p.: 205-207 ${ }^{\circ} \mathrm{C}$; MS (ESI) m/z: $483.4[\mathrm{M}+\mathrm{H}]^{+}$; IR $\left(\mathrm{KBr}, \mathrm{cm}^{-1}\right): 1618.3 ;{ }^{1} \mathrm{H}$ NMR (600 MHz, DMSO- $\left.d_{6}\right) \delta 11.13$ (s, 1H, -NH-), 10.43 (s, 1H, -NH-), 8.30-8.24 (m, 2H, -CH=, indole- $\mathrm{H}), 8.14-8.05(\mathrm{~m}, 1 \mathrm{H}$, indole- $\mathrm{H}), 7.88(\mathrm{~s}, 1 \mathrm{H}$, indole- $\mathrm{H}), 7.68(\mathrm{~d}, J=7.8 \mathrm{~Hz}, 1 \mathrm{H}$, benzothiazole-H), $7.54(\mathrm{~d}, J=7.8 \mathrm{~Hz}, 1 \mathrm{H}$, benzothiazole- $\mathrm{H}), 7.37(\mathrm{~d}, J=7.8 \mathrm{~Hz}, 1 \mathrm{H}$, benzothiazole-H), 7.35-7.31 (m, 2H, 2-indole-H), 7.29-7.21 (m, 5H, 5-benzyl-H), 5.48 (s, 2H, $-\mathrm{CH}_{2^{-}}$), $3.64\left(\mathrm{~s}, 2 \mathrm{H},-\mathrm{CH}_{2^{-}}\right), 2.28\left(\mathrm{~s}, 6 \mathrm{H}, 2-\mathrm{CH}_{3}\right) ;{ }^{13} \mathrm{C}$ NMR (100 MHz, DMSO-d $\left.d_{6}\right) \delta 159.57$, 152.59, 148.30, 141.13, 137.96, 137.31, 134.10, 133.67, 132.11, 129.10, 128.03, 127.60, 127.44, 125.38, 123.29, 122.21, 121.91, 121.29, 119.80, 111.33, 111.19, 63.62, 49.86, 45.27; Anal. Calcd, for $\mathrm{C}_{27} \mathrm{H}_{26} \mathrm{~N}_{6} \mathrm{OS}(\%): \mathrm{C}, 67.20 ; \mathrm{H}, 5.43 ; \mathrm{N}, 17.41$. Found (\%):C, 67.12; H, 5.33; N, 17.33.

5.1.8.10.(E)-4-(6-((dimethylamino)methyl)benzothiazol-2-yl)-1-((1-(4-methylbenzyl)-1H-indol-3-yl 
)methylene)semicarbazide (20b)

Yield: 64\%; M.p.: $175-177{ }^{\circ} \mathrm{C}$; MS (ESI) m/z: $497.4[\mathrm{M}+\mathrm{H}]^{+}$; IR $\left(\mathrm{KBr}, \mathrm{cm}^{-1}\right): 1617.7 ;{ }^{1} \mathrm{H}$ NMR (400 MHz, DMSO- $\left.d_{6}\right) \delta 11.06$ (s, 1H, -NH-), 10.46 (s, 1H, -NH-), 8.26 (s, 1H, -CH=), 8.25 $(\mathrm{d}, J=4.0 \mathrm{~Hz}, 1 \mathrm{H}$, indole-H), 8.11-8.02 (m, 1H, indole-H), $7.88(\mathrm{~s}, 1 \mathrm{H}$, indole- $\mathrm{H}), 7.68(\mathrm{~d}, J=8.2$ $\mathrm{Hz}, 1 \mathrm{H}$, benzothiazole- $\mathrm{H}), 7.56-7.50(\mathrm{~m}, 1 \mathrm{H}$, benzothiazole- $\mathrm{H}), 7.37(\mathrm{~d}, J=8.2 \mathrm{~Hz}, 1 \mathrm{H}$, benzothiazole-H), 7.25-7.20 (m, 2H, 2-indole-H), 7.18 (d, $J=8.0 \mathrm{~Hz}, 2 \mathrm{H}, 2$-benzyl-H), 7.13 (d, $J$ $=8.0 \mathrm{~Hz}, 2 \mathrm{H}, 2$-benzyl-H), $5.41\left(\mathrm{~s}, 2 \mathrm{H},-\mathrm{CH}_{2}-\right), 3.66\left(\mathrm{~s}, 2 \mathrm{H},-\mathrm{CH}_{2}-\right), 2.29\left(\mathrm{~s}, 6 \mathrm{H}, 2-\mathrm{CH}_{3}\right), 2.25(\mathrm{~s}$, $\left.3 \mathrm{H},-\mathrm{CH}_{3}\right) ;{ }^{13} \mathrm{C}$ NMR $\left(100 \mathrm{MHz}, \mathrm{DMSO}-d_{6}\right) \delta 159.63,152.57,148.40,141.20,137.26,137.25$, 134.87, 133.56, 132.12, 129.63, 127.66, 127.54, 125.40, 123.22, 122.21, 122.06, 121.24, 119.84, 119.79, 111.30, 111.20, 63.40, 49.68, 45.06, 21.68; Anal. Calcd, for $\mathrm{C}_{28} \mathrm{H}_{28} \mathrm{~N}_{6} \mathrm{OS}(\%): \mathrm{C}, 67.72 ; \mathrm{H}$, $5.68 ; \mathrm{N}, 16.92$. Found (\%):C, 67.82; H, 5.55; N, 16.99 .

5.1.8.11.(E)-4-(6-((dimethylamino)methyl)benzothiazol-2-yl)-1-((1-(4-tert-butylbenzyl)-1H-indol-3 -yl)methylene)semicarbazide (20c)

Yield: 71\%; M.p.: $150-152{ }^{\circ} \mathrm{C}$; MS (ESI) m/z: $539.4[\mathrm{M}+\mathrm{H}]^{+}$; IR $\left(\mathrm{KBr}, \mathrm{cm}^{-1}\right): 1605.5 ;{ }^{1} \mathrm{H}$ NMR (400 MHz, DMSO- $\left.d_{6}\right) \delta 11.06$ (s, 1H, -NH-), 10.42 (s, 1H, -NH-), 8.26 (s, 1H, -CH=), 8.25 (d, $J=2.4 \mathrm{~Hz}, 1 \mathrm{H}$, indole-H), 8.11-8.04 (m, 1H, indole-H), 7.84 (s, $1 \mathrm{H}$, indole-H), 7.65 (d, $J=8.0$ $\mathrm{Hz}, 1 \mathrm{H}$, benzothiazole- $\mathrm{H}), 7.60-7.54(\mathrm{~m}, 1 \mathrm{H}$, benzothiazole- $\mathrm{H}), 7.34(\mathrm{~d}, J=8.4 \mathrm{~Hz}, 3 \mathrm{H}$, benzothiazole-H, 2-benzyl-H), 7.26-7.22 (m, 2H, 2-indole- $\mathrm{H}), 7.20(\mathrm{~d}, J=8.4 \mathrm{~Hz}, 2 \mathrm{H}$, 2-benzyl-H), 5.42 (s, 2H, - $\left.\mathrm{CH}_{2}-\right), 3.53$ (s, 2H, - $\left.\mathrm{CH}_{2}-\right), 2.20\left(\mathrm{~s}, 6 \mathrm{H}, 2-\mathrm{CH}_{3}\right), 1.23$ (s, 9H, 3- $\mathrm{CH}_{3}$ ); ${ }^{13} \mathrm{C}$ NMR (100 MHz, DMSO- $\left.d_{6}\right) \delta 159.57,152.58,150.46,148.34,141.13,137.30,134.99$, 133.95, 133.60, 132.11, 127.46, 127.39, 125.83, 125.36, 123.26, 122.18, 121.95, 121.25, 119.81, 111.27, 111.20, 63.55, 49.50, 45.21, 34.65, 31.51; Anal. Calcd, for $\mathrm{C}_{31} \mathrm{H}_{34} \mathrm{~N}_{6} \mathrm{OS}(\%): \mathrm{C}, 69.12 ; \mathrm{H}$, 6.36; N, 15.60. Found (\%):C, 69.02; H, 6.46; N, 15.68.

5.1.8.12.(E)-4-(6-((dimethylamino)methyl)benzothiazol-2-yl)-1-((1-(4-chlorobenzyl)-1H-indol-3-yl )methylene) semicarbazide (20d)

Yield: 68\%; M.p.: 188-190 ${ }^{\circ} \mathrm{C}$; MS (ESI) m/z: $517.3[\mathrm{M}+\mathrm{H}]^{+}$; IR $\left(\mathrm{KBr}, \mathrm{cm}^{-1}\right): 1615.8 ;{ }^{1} \mathrm{H}$ NMR (600 MHz, DMSO- $\left.d_{6}\right) \delta 11.11$ (s, 1H, -NH-), 10.42 (s, 1H, -NH-), 8.30-8.23 (m, 2H, -CH=, indole- $\mathrm{H}), 8.11-8.04(\mathrm{~m}, 1 \mathrm{H}$, indole- $\mathrm{H}), 7.86(\mathrm{~s}, 1 \mathrm{H}$, indole- $\mathrm{H}), 7.67(\mathrm{~d}, J=7.8 \mathrm{~Hz}, 1 \mathrm{H}$, benzothiazole-H), 7.55-7.50 (m, 1H, benzothiazole-H), 7.40 (d, $J=8.4 \mathrm{~Hz}, 2 \mathrm{H}, 2$-benzyl-H), 7.36 (d, $J=7.8 \mathrm{~Hz}, 1 \mathrm{H}$, benzothiazole-H), $7.28(\mathrm{~d}, J=8.4 \mathrm{~Hz}, 2 \mathrm{H}, 2$-benzyl-H), 7.26-7.21 (m, 2H, indole- $\mathrm{H}), 5.48\left(\mathrm{~s}, 2 \mathrm{H},-\mathrm{CH}_{2}-\right), 3.58\left(\mathrm{~s}, 2 \mathrm{H},-\mathrm{CH}_{2}-\right), 2.24\left(\mathrm{~s}, 6 \mathrm{H}, 2-\mathrm{CH}_{3}\right)$; Anal. Calcd, for $\mathrm{C}_{27} \mathrm{H}_{25} \mathrm{ClN}_{6} \mathrm{OS}(\%): \mathrm{C}, 62.72 ; \mathrm{H}, 4.87 ; \mathrm{N}, 16.25$. Found (\%):C, 62.81; H, 4.94; N, 16.35 .

5.1.8.13.(E)-4-(6-((dimethylamino)methyl)benzothiazol-2-yl)-1-((1-(3-chlorobenzyl)-1H-indol-3-yl methylene)semicarbazide (20e)

Yield: 71\%; M.p.: 162-164 ${ }^{\circ} \mathrm{C}$; MS (ESI) m/z: $517.0[\mathrm{M}+\mathrm{H}]^{+} ;{ }^{1} \mathrm{H}$ NMR $\left(600 \mathrm{MHz}, \mathrm{DMSO}-d_{6}\right)$ $\delta 11.03$ (s, 1H, -NH-), 10.41 (s, 1H, -NH-), 8.26 (s, 1H, -CH=), 8.25 (d, $J=1.8 \mathrm{~Hz}, 1 \mathrm{H}$, indole-H), 8.11-8.03 (m, 1H, indole- $\mathrm{H}), 7.81(\mathrm{~s}, 1 \mathrm{H}$, indole- $\mathrm{H}), 7.63(\mathrm{~d}, J=7.2 \mathrm{~Hz}, 1 \mathrm{H}$, benzothiazole- $\mathrm{H})$, 7.56-7.52 (m, 1H, benzothiazole-H), 7.37-7.28 (m, 4H, benzothiazole- $\mathrm{H}, 3$-benzyl-H), 7.26-7.21 (m, 2H, indole-H), 7.19 (d, $J=7.2 \mathrm{~Hz}, 1 \mathrm{H}$, benzyl-H), $5.48\left(\mathrm{~s}, 2 \mathrm{H},-\mathrm{CH}_{2}-\right), 3.46\left(\mathrm{~s}, 2 \mathrm{H},-\mathrm{CH}_{2}-\right)$, 2.15 (s, 6H, 2- $\left.\mathrm{CH}_{3}\right)$; Anal. Calcd, for $\mathrm{C}_{27} \mathrm{H}_{25} \mathrm{ClN}_{6} \mathrm{OS}(\%): \mathrm{C}, 62.72 ; \mathrm{H}, 4.87 ; \mathrm{N}, 16.25$. Found $(\%): \mathrm{C}, 62.77 ; \mathrm{H}, 4.84 ; \mathrm{N}, 16.32$.

5.1.8.14.(E)-4-(6-((dimethylamino)methyl)benzothiazol-2-yl)-1-((1-(2-chlorobenzyl)-1H-indol-3-yl methylene)semicarbazide (20f)

Yield: 59\%; M.p.: 204-206 ${ }^{\circ}$ C; MS (ESI) m/z: $517.3[\mathrm{M}+\mathrm{H}]^{+} ;{ }^{1} \mathrm{H}$ NMR $\left(600 \mathrm{MHz}\right.$, DMSO- $\left.d_{6}\right)$ 
$\delta 11.11(\mathrm{~s}, 1 \mathrm{H},-\mathrm{NH}-), 10.44(\mathrm{~s}, 1 \mathrm{H},-\mathrm{NH}-), 8.33-8.29(\mathrm{~m}, 1 \mathrm{H}$, indole-H), 8.27 (s, 1H, -CH=), 8.07-7.92 (m, 1H, indole- $\mathrm{H}), 7.84(\mathrm{~s}, 1 \mathrm{H}$, indole-H), $7.65(\mathrm{~d}, J=7.2 \mathrm{~Hz}, 1 \mathrm{H}$, benzothiazole- $\mathrm{H})$, $7.54(\mathrm{dd}, J=7.2,0.6 \mathrm{~Hz}, 1 \mathrm{H}$, benzothiazole-H), 7.51-7.44 (m, 1H, benzothiazole-H), $7.34(\mathrm{~m}, 2 \mathrm{H}$, indole-H), $7.26\left(\mathrm{~m}, 3 \mathrm{H}, 3\right.$-benzyl-H), $6.81\left(\mathrm{~d}, J=7.2 \mathrm{~Hz}, 1 \mathrm{H}\right.$, benzyl-H), $5.58\left(\mathrm{~s}, 2 \mathrm{H},-\mathrm{CH}_{2}{ }^{-}\right), 3.53$ (s, $\left.2 \mathrm{H},-\mathrm{CH}_{2}-\right), 2.21$ (s, 6H, 2- $\left.\mathrm{CH}_{3}\right) ;{ }^{13} \mathrm{C}$ NMR (100 MHz, DMSO-d $\left.d_{6}\right) \delta 159.58,152.60,148.26$, $141.05,137.45,135.12,134.12,133.75,132.41,132.08,130.05,129.94,129.10,128.08,127.44$, 125.27, 123.52, 122.36, 121.91, 121.48, 119.80, 111.63, 110.98, 63.62, 47.69, 45.28; Anal. Calcd, for $\mathrm{C}_{27} \mathrm{H}_{25} \mathrm{ClN}_{6} \mathrm{OS}(\%): \mathrm{C}, 62.72 ; \mathrm{H}, 4.87 ; \mathrm{N}, 16.25$. Found (\%):C, 62.87; H, 4.72; N, 16.22.

5.1.8.15.(E)-4-(6-((dimethylamino)methyl)benzothiazol-2-yl)-1-((1-(3,4-dichlorobenzyl)-1H-indol3-yl)methylene) semicarbazide (20g)

Yield: 55\%; M.p.: 190-192 ${ }^{\circ} \mathrm{C}$; MS (ESI) m/z: $551.2[\mathrm{M}+\mathrm{H}]^{+} ;{ }^{1} \mathrm{H}$ NMR (400 MHz, DMSO- $\left.d_{6}\right)$ $\delta 11.00(\mathrm{~s}, 1 \mathrm{H},-\mathrm{NH}-), 10.48(\mathrm{~s}, 1 \mathrm{H},-\mathrm{NH}-), 8.34-8.29(\mathrm{~m}, 1 \mathrm{H}$, indole-H), 8.28 (s, 1H, -CH=), 8.08-7.93 (m, 1H, indole- $\mathrm{H}), 7.83(\mathrm{~s}, 1 \mathrm{H}$, indole- $\mathrm{H}), 7.64(\mathrm{~d}, J=8.0 \mathrm{~Hz}, 1 \mathrm{H}$, benzothiazole- $\mathrm{H})$, $7.60(\mathrm{~d}, J=8.0 \mathrm{~Hz}, 1 \mathrm{H}$, benzothiazole-H), 7.51-7.45 (m, 1H, benzothiazole-H), $7.33(\mathrm{~d}, J=8.4 \mathrm{~Hz}$, 1H, benzyl-H), 7.31-7.22 (m, 3H, benzyl-H, 2-indole-H), $6.66(\mathrm{~d}, J=8.4 \mathrm{~Hz}, 1 \mathrm{H}$, benzyl-H), 5.62 (s, $2 \mathrm{H},-\mathrm{CH}_{2}$ ), 3.47 (s, $2 \mathrm{H},-\mathrm{CH}_{2}$ ), 2.17 (s, $6 \mathrm{H}, 2-\mathrm{CH}_{3}$ ); Anal. Calcd, for $\mathrm{C}_{27} \mathrm{H}_{24} \mathrm{Cl}_{2} \mathrm{~N}_{6} \mathrm{OS}(\%): \mathrm{C}$, 58.80; H, 4.39; N, 15.24. Found (\%):C, 58.89; H, 4.49; N, 15.31 .

5.1.8.16.(E)-4-(6-((dimethylamino)methyl)benzothiazol-2-yl)-1-((1-(2,4-dichlorobenzyl)-1H-indol3-yl)methylene) semicarbazide (20h)

Yield: 67\%; M.p.: $138-141{ }^{\circ} \mathrm{C}$; MS (ESI) m/z: $551.0[\mathrm{M}+\mathrm{H}]^{+} ;{ }^{1} \mathrm{H}$ NMR (400 MHz, DMSO- $\left.d_{6}\right)$ $\delta 11.11(\mathrm{~s}, 1 \mathrm{H},-\mathrm{NH}-), 10.46(\mathrm{~s}, 1 \mathrm{H},-\mathrm{NH}-), 8.34-8.30(\mathrm{~m}, 1 \mathrm{H}$, indole-H), $8.28(\mathrm{~s}, 1 \mathrm{H},-\mathrm{CH}=)$, 8.05-7.90 (m, 2H, 2-indole-H), $7.72(\mathrm{~d}, J=2.0 \mathrm{~Hz}, 2 \mathrm{H}$, benzothiazole- $\mathrm{H}), 7.51-7.40(\mathrm{~m}, 2 \mathrm{H}$, benzothiazole-H, benzyl-H), 7.37 (dd, $J=8.4,2.0 \mathrm{~Hz}, 1 \mathrm{H}$, benzothiazole- $\mathrm{H}), 7.31-7.20(\mathrm{~m}, 2 \mathrm{H}$, 2-indole-H), $6.80\left(\mathrm{~d}, J=8.4 \mathrm{~Hz}, 1 \mathrm{H}\right.$, benzyl-H), $5.56\left(\mathrm{~s}, 2 \mathrm{H},-\mathrm{CH}_{2}-\right), 3.95\left(\mathrm{~s}, 2 \mathrm{H},-\mathrm{CH}_{2}-\right), 2.48(\mathrm{~s}$, $\left.6 \mathrm{H}, 2-\mathrm{CH}_{3}\right)$; Anal. Calcd, for $\mathrm{C}_{27} \mathrm{H}_{24} \mathrm{Cl}_{2} \mathrm{~N}_{6} \mathrm{OS}(\%): \mathrm{C}, 58.80 ; \mathrm{H}, 4.39 ; \mathrm{N}, 15.24$. Found (\%):C, $58.90 ; \mathrm{H}, 4.31 ; \mathrm{N}, 15.29$.

5.1.8.17.(E)-4-(6-((diethylamino)methyl)benzothiazol-2-yl)-1-((1-benzyl-1H-indol-3-yl)methylene) semicarbazide (20i)

Yield: 58\%; M.p.: $195-197{ }^{\circ} \mathrm{C}$; MS (ESI) m/z: $511.3[\mathrm{M}+\mathrm{H}]^{+} ;{ }^{1} \mathrm{H}$ NMR (400 MHz, DMSO- $\left.d_{6}\right)$ $\delta 11.05$ (s, 1H, -NH-), 10.44 (s, 1H, -NH-), 8.27 (s, 1H, -CH=), 8.27-8.23 (m, 1H, indole-H), 8.08 (s, $1 \mathrm{H}$, indole- $\mathrm{H}), 7.88(\mathrm{~s}, 1 \mathrm{H}$, indole- $\mathrm{H}), 7.66(\mathrm{~d}, J=7.8 \mathrm{~Hz}, 1 \mathrm{H}$, benzothiazole- $\mathrm{H}), 7.58-7.50(\mathrm{~m}$, $1 \mathrm{H}$, benzothiazole- $\mathrm{H})$, 7.44-7.30 (m, 3H, benzothiazole- $\mathrm{H}, 2$-indole- $\mathrm{H}), 7.30-7.17(\mathrm{~m}, 5 \mathrm{H}$, 5-benzyl-H), 5.48 (s, 2H, - $\mathrm{CH}_{2^{-}}$), 3.65 (s, 2H, - $\mathrm{CH}_{2^{-}}$), 3.34 (s, 4H, 2- $\left.\mathrm{CH}_{2}-\right), 1.03\left(\mathrm{~s}, 6 \mathrm{H}, 2-\mathrm{CH}_{3}\right)$; Anal. Calcd, for $\mathrm{C}_{29} \mathrm{H}_{30} \mathrm{~N}_{6} \mathrm{OS}(\%): \mathrm{C}, 68.21 ; \mathrm{H}, 5.92 ; \mathrm{N}, 16.46$. Found (\%):C, 68.29; H, 5.82; N, 16.57 .

5.1.8.18.(E)-4-(6-((diethylamino)methyl)benzothiazol-2-yl)-1-((1-(4-methylbenzyl)-1H-indol-3-yl) methylene)semicarbazide (20j)

Yield: 54\%; M.p.: $194-197^{\circ} \mathrm{C}$; MS (ESI) m/z: $525.0[\mathrm{M}+\mathrm{H}]^{+}$; $\left.1 \mathrm{H} \mathrm{NMR} \mathrm{(400} \mathrm{MHz,} \mathrm{CDCl}_{3}\right) \delta$ 9.52 (s, 2H, 2-NH-), 8.23-8.17 (m, 1H, indole-H), 8.17 (s, 1H, - $\mathrm{CH}=), 7.90$ (s, 1H, indole-H), 7.74 (d, $J=8.2 \mathrm{~Hz}, 1 \mathrm{H}$, benzothiazole-H), $7.44(\mathrm{~s}, 1 \mathrm{H}$, benzothiazole-H), $7.40(\mathrm{~d}, J=8.2 \mathrm{~Hz}, 1 \mathrm{H}$, benzothiazole-H), 7.35-7.30 (m, 3H, 3-indole-H), 7.13 (d, $J=8.0 \mathrm{~Hz}, 2 \mathrm{H}, 2$-benzyl-H), 7.06 (d, $J$ $=8.0 \mathrm{~Hz}, 2 \mathrm{H}, 2$-benzyl- $\mathrm{H}), 5.27\left(\mathrm{~s}, 2 \mathrm{H},-\mathrm{CH}_{2}-\right), 3.83\left(\mathrm{~s}, 2 \mathrm{H},-\mathrm{CH}_{2}-\right), 2.74-2.60\left(\mathrm{~m}, 4 \mathrm{H}, 2-\mathrm{CH}_{2}-\right)$, 2.32 (s, $\left.3 \mathrm{H},-\mathrm{CH}_{3}\right), 1.15$ (t, $J=7.2 \mathrm{~Hz}, 6 \mathrm{H}, 2-\mathrm{CH}_{3}$ ); Anal. Calcd, for $\mathrm{C}_{30} \mathrm{H}_{32} \mathrm{~N}_{6} \mathrm{OS}(\%): \mathrm{C}, 68.67 ; \mathrm{H}$, 6.15; N, 16.02. Found (\%):C, 68.75; H, 6.24; N, 16.12. 
5.1.8.19.(E)-4-(6-((diethylamino)methyl)benzothiazol-2-yl)-1-((1-(4-tert-butylbenzyl)-1H-indol-3yl)methylene) semicarbazide (20k)

Yield: 64\%; M.p.: $184-186{ }^{\circ} \mathrm{C}$; MS (ESI) m/z: $567.4[\mathrm{M}+\mathrm{H}]^{+} ;{ }^{1} \mathrm{H}$ NMR $(400 \mathrm{MHz}$, DMSO- $\left.\left.d_{6}\right)\right) \delta 11.04(\mathrm{~s}, 1 \mathrm{H},-\mathrm{NH}-), 10.48(\mathrm{~s}, 1 \mathrm{H},-\mathrm{NH}-), 8.28(\mathrm{~s}, 1 \mathrm{H},-\mathrm{CH}=), 8.27-8.22(\mathrm{~m}, 1 \mathrm{H}$, indole- $\mathrm{H}), 8.11(\mathrm{~s}, 1 \mathrm{H}$, indole- $\mathrm{H}), 8.09-8.04(\mathrm{~m}, 1 \mathrm{H}$, benzothiazole- $\mathrm{H}), 7.80(\mathrm{~d}, J=8.0 \mathrm{~Hz}, 1 \mathrm{H}$, benzothiazole-H), 7.57 (t, $J=8.0 \mathrm{~Hz}, 2 \mathrm{H}, 2$-benzyl-H), 7.34 (d, $J=8.4 \mathrm{~Hz}, 2 \mathrm{H}, 2$-benzyl-H), 7.29-7.14 (m, 4H, 3-indole- $\mathrm{H}$, benzothiazole- $\mathrm{H}), 5.43\left(\mathrm{~s}, 2 \mathrm{H},-\mathrm{CH}_{2}-\right), 4.39\left(\mathrm{~s}, 2 \mathrm{H},-\mathrm{CH}_{2}-\right)$, 3.18-3.04 (m, 4H, 2- $\left.\mathrm{CH}_{2}-\right)$, 1.34-1.13 (m, $\left.15 \mathrm{H}, 5-\mathrm{CH}_{3}\right)$; Anal. Calcd, for $\mathrm{C}_{33} \mathrm{H}_{38} \mathrm{~N}_{6} \mathrm{OS}(\%): \mathrm{C}$, 69.93; H, 6.76; N, 14.83. Found (\%):C, 69.84; H, 6.68; N, 14.89.

5.1.8.20.(E)-4-(6-((diethylamino)methyl)benzothiazol-2-yl)-1-((1-(4-chlorobenzyl)-1H-indol-3-yl) methylene)semicarbazide (20l)

Yield: 53\%; M.p.: $173-175{ }^{\circ} \mathrm{C}$; MS (ESI) m/z: $545.3[\mathrm{M}+\mathrm{H}]^{+}$; IR $\left(\mathrm{KBr}, \mathrm{cm}^{-1}\right): 1614.0 ;{ }^{1} \mathrm{H}$ NMR (600 MHz, DMSO- $\left.\left.d_{6}\right)\right) \delta 11.13(\mathrm{~s}, 1 \mathrm{H},-\mathrm{NH}-), 10.47$ (s, 1H, -NH-), 8.28 (s, 1H, -CH=), 8.27 $(\mathrm{d}, J=1.6 \mathrm{~Hz}, 1 \mathrm{H}$, indole-H), $8.08(\mathrm{~s}, 1 \mathrm{H}$, indole-H), 8.02-7.89 (s, $1 \mathrm{H}$, benzothiazole- $\mathrm{H}), 7.70(\mathrm{~s}$, $1 \mathrm{H}$, benzothiazole-H), 7.55-7.51 (m, 1H, benzothiazole- $\mathrm{H}), 7.40(\mathrm{~d}, J=8.4 \mathrm{~Hz}, 2 \mathrm{H}, 2-$ benzyl-H), 7.28 (d, $J=8.4 \mathrm{~Hz}, 2 \mathrm{H}, 2$-benzyl-H), 7.27-7.20 (m, 3H, 3-indole-H), 5.48 (s, 2H, - $\left.\mathrm{CH}_{2}-\right), 3.35$ (s, $\left.4 \mathrm{H}, 2-\mathrm{CH}_{2}-\right), 2.79\left(\mathrm{~s}, 2 \mathrm{H},-\mathrm{CH}_{2}-\right), 1.11\left(\mathrm{~s}, 6 \mathrm{H}, 2-\mathrm{CH}_{3}\right)$; Anal. Calcd, for $\mathrm{C}_{29} \mathrm{H}_{29} \mathrm{ClN}_{6} \mathrm{OS}(\%): \mathrm{C}$, 63.90; H, 5.36; N, 15.42. Found (\%):C, 63.81; H, 5.47; N, 15.34.

5.1.8.21.(E)-4-(6-((diethylamino)methyl)benzothiazol-2-yl)-1-((1-(3-chlorobenzyl)-1H-indol-3-yl) methylene) semicarbazide (20m)

Yield: 61\%; M.p.: 210-212 ${ }^{\circ} \mathrm{C}$; MS (ESI) m/z: $545.0[\mathrm{M}+\mathrm{H}]^{+}$; ${ }^{1} \mathrm{H}$ NMR $(400 \mathrm{MHz}$, DMSO- $\left.\left.d_{6}\right)\right) \delta 11.11(\mathrm{~s}, 1 \mathrm{H},-\mathrm{NH}-), 10.65(\mathrm{~s}, 1 \mathrm{H},-\mathrm{NH}-), 8.30$ (s, 1H, - $\left.\mathrm{CH}=\right), 8.29-8.27(\mathrm{~m}, 1 \mathrm{H}$, indole-H), 8.18-8.07 (m, 2H, indole-H, benzothiazole-H), 7.80 (s, 1H, benzothiazole-H), 7.63-7.54 $(\mathrm{m}, 2 \mathrm{H}$, benzothiazole- $\mathrm{H}$, benzyl- $\mathrm{H}), 7.40-7.33(\mathrm{~m}, 3 \mathrm{H}, 3$-indole- $\mathrm{H}), 7.30-7.20(\mathrm{~m}, 3 \mathrm{H}$, 3-benzyl-H), 5.51 (s, 2H, - $\mathrm{CH}_{2}$ ), 4.39 (s, 2H, - $\mathrm{CH}_{2}$ ), 3.10 (s, 4H, 2- $\mathrm{CH}_{2^{-}}$), 1.27 (t, J=7.2 Hz, 6H, 2- $\mathrm{CH}_{3}$ ); Anal. Calcd, for $\mathrm{C}_{29} \mathrm{H}_{29} \mathrm{ClN}_{6} \mathrm{OS}(\%): \mathrm{C}, 63.90 ; \mathrm{H}, 5.36 ; \mathrm{N}, 15.42$. Found (\%):C, 63.96; H, 5.27; N, 15.37 .

5.1.8.22.(E)-4-(6-((diethylamino)methyl)benzothiazol-2-yl)-1-((1-(2-chlorobenzyl)-1H-indol-3-yl) methylene)semicarbazide (20n)

Yield: 53\%; M.p.: 202-204 ${ }^{\circ} \mathrm{C}$; MS (ESI) m/z: $545.3[\mathrm{M}+\mathrm{H}]^{+}$; ${ }^{1} \mathrm{H}$ NMR $(400 \mathrm{MHz}$, DMSO- $\left.\left.d_{6}\right)\right) \delta 11.10(\mathrm{~s}, 1 \mathrm{H},-\mathrm{NH}-), 10.53(\mathrm{~s}, 1 \mathrm{H},-\mathrm{NH}-), 8.33-8.29(\mathrm{~m}, 1 \mathrm{H}$, indole-H), $8.28(\mathrm{~s}, 1 \mathrm{H}$, - $\mathrm{CH}=$ ), 8.01-7.94 (m, 2H, 2-indole-H), 7.76-7.68 (m, 1H, benzothiazole-H), 7.54 (dd, $J=8.0,1.6$ $\mathrm{Hz}, 1 \mathrm{H}$, benzothiazole- $\mathrm{H}), 7.51-7.44(\mathrm{~m}, 2 \mathrm{H}, 2$-indole- $\mathrm{H}), 7.34(\mathrm{td}, J=8.0,1.6 \mathrm{~Hz}, 1 \mathrm{H}$, benzothiazole-H), 7.30-7.21 (m, 3H, 3-benzyl-H), $6.82(\mathrm{~d}, J=6.8 \mathrm{~Hz}, 1 \mathrm{H}$, benzyl-H), $5.58(\mathrm{~s}, 2 \mathrm{H}$, $\left.-\mathrm{CH}_{2^{-}}\right), 4.26\left(\mathrm{~s}, 2 \mathrm{H},-\mathrm{CH}_{2^{-}}\right), 3.24-2.53\left(\mathrm{~m}, 4 \mathrm{H}, 2-\mathrm{CH}_{2^{-}}\right), 1.49-0.81\left(\mathrm{~m}, 6 \mathrm{H}, 2-\mathrm{CH}_{3}\right)$; Anal. Calcd, for $\mathrm{C}_{29} \mathrm{H}_{29} \mathrm{ClN}_{6} \mathrm{OS}(\%): \mathrm{C}, 63.90 ; \mathrm{H}, 5.36 ; \mathrm{N}, 15.42$. Found (\%):C, 63.98; H, 5.41; N, 15.32 .

5.1.8.23.(E)-4-(6-((diethylamino)methyl)benzothiazol-2-yl)-1-((1-(3,4-dichlorobenzyl)-1H-indol-3 -yl)methylene)semicarbazide (20o)

Yield: 64\%; M.p.: 199-201 ${ }^{\circ} \mathrm{C}$; MS (ESI) m/z: $579.1[\mathrm{M}+\mathrm{H}]^{+}$; ${ }^{1} \mathrm{H}$ NMR (400 MHz, DMSO- $\left.\left.d_{6}\right)\right) \delta 11.13(\mathrm{~s}, 1 \mathrm{H},-\mathrm{NH}-), 9.26(\mathrm{~s}, 1 \mathrm{H},-\mathrm{NH}-), 8.33(\mathrm{dd}, J=6.1,3.0 \mathrm{~Hz}, 1 \mathrm{H}$, indole-H), 8.36-8.31 (m, 1H, - $\mathrm{CH}=), 8.11(\mathrm{~s}, 1 \mathrm{H}$, indole- $\mathrm{H}), 8.00(\mathrm{~s}, 1 \mathrm{H}$, indole- $\mathrm{H}), 7.79(\mathrm{~d}, J=8.0 \mathrm{~Hz}, 1 \mathrm{H}$, benzothiazole-H), $7.61(\mathrm{~d}, J=8.0 \mathrm{~Hz}, 1 \mathrm{H}$, benzothiazole-H), $7.56(\mathrm{~d}, J=8.0 \mathrm{~Hz}, 1 \mathrm{H}$, benzothiazole- $\mathrm{H})$, 7.52-7.46 (m, 1H, indole- $\mathrm{H}), 7.34-7.22(\mathrm{~m}, 3 \mathrm{H}$, indole- $\mathrm{H}, 2$-benzyl-H), 6.67 (d, $J=8.0 \mathrm{~Hz}, 1 \mathrm{H}$, benzyl- $\mathrm{H}), 5.63\left(\mathrm{~s}, 2 \mathrm{H},-\mathrm{CH}_{2^{-}}\right), 4.41\left(\mathrm{~s}, 2 \mathrm{H},-\mathrm{CH}_{2}-\right), 3.21-3.03\left(\mathrm{~m}, 4 \mathrm{H}, 2-\mathrm{CH}_{2}{ }^{-}\right)$, 
$1.26\left(\mathrm{t}, J=7.2 \mathrm{~Hz}, 6 \mathrm{H}, 2-\mathrm{CH}_{3}\right)$; Anal. Calcd, for $\mathrm{C}_{29} \mathrm{H}_{28} \mathrm{Cl}_{2} \mathrm{~N} 6 \mathrm{OS}(\%): \mathrm{C}, 60.10 ; \mathrm{H}, 4.87 ; \mathrm{N}, 14.50$. Found (\%):C, 60.19; H, 4.77; N, 14.58 .

5.1.8.24.(E)-4-(6-((diethylamino)methyl)benzothiazol-2-yl)-1-((1-(2,4-dichlorobenzyl)-1H-indol-3 -yl)methylene)semicarbazide (20p)

Yield: 58\%; M.p.: $142-145{ }^{\circ} \mathrm{C}$; MS (ESI) m/z: $579.1[\mathrm{M}+\mathrm{H}]^{+}$; ${ }^{1} \mathrm{H}$ NMR $(400 \mathrm{MHz}$, DMSO- $\left.\left.d_{6}\right)\right) \delta 11.08(\mathrm{~s}, 1 \mathrm{H},-\mathrm{NH}-), 10.51(\mathrm{~s}, 1 \mathrm{H},-\mathrm{NH}-), 8.34-8.29(\mathrm{~m}, 1 \mathrm{H}$, indole- $\mathrm{H}), 8.27(\mathrm{~s}, 1 \mathrm{H}$, - $\mathrm{CH}=)$, 8.07-7.85 (s, 2H, 2-indole-H), $7.72(\mathrm{~d}, J=2.0 \mathrm{~Hz}, 1 \mathrm{H}$, benzothiazole-H), 7.69 (d, $J=4.0$ $\mathrm{Hz}, 1 \mathrm{H}$, benzothiazole-H), 7.53-7.40 (m, 2H, 2-indole-H), 7.37 (dd, $J=8.4,2.0 \mathrm{~Hz}, 1 \mathrm{H}$, benzothiazole-H), 7.31-7.19 (m, 2H, 2-benzyl-H), 6.80 (d, $J=8.4 \mathrm{~Hz}, 1 \mathrm{H}$, benzyl-H), $5.56(\mathrm{~s}, 2 \mathrm{H}$, $-\mathrm{CH}_{2-}$ ), 3.65 (s, $\left.2 \mathrm{H},-\mathrm{CH}_{2}-\right), 3.01-2.51\left(\mathrm{~m}, 4 \mathrm{H}, 2-\mathrm{CH}_{2}-\right), 1.09$ (s, 6H, 2- $\left.\mathrm{CH}_{3}\right)$; Anal. Calcd, for $\mathrm{C}_{29} \mathrm{H}_{28} \mathrm{Cl}_{2} \mathrm{~N} 6 \mathrm{OS}(\%): \mathrm{C}, 60.10 ; \mathrm{H}, 4.87 ; \mathrm{N}, 14.50$. Found (\%):C, 60.15; H, 4.90; N, 14.48.

5.1.8.25.(E)-4-(6-((4-methylpiperidin-1-yl)methyl)benzothiazol-2-yl)-1-((1-benzyl-1H-indol-3-yl)m ethylene) semicarbazide (20q)

Yield: 61\%; M.p.: $218-220{ }^{\circ} \mathrm{C}$; MS (ESI) m/z: $537.4[\mathrm{M}+\mathrm{H}]^{+}$; IR $\left(\mathrm{KBr}, \mathrm{cm}^{-1}\right): 1617.9 ;{ }^{1} \mathrm{H}$ NMR (400 MHz, DMSO- $\left.\left.d_{6}\right)\right) \delta 11.07(\mathrm{~s}, 1 \mathrm{H},-\mathrm{NH}-), 10.44(\mathrm{~s}, 1 \mathrm{H},-\mathrm{NH}-), 8.27(\mathrm{~s}, 1 \mathrm{H},-\mathrm{CH}=)$, 8.26-8.23 (m, 1H, indole-H), 8.14-8.03 (m, 1H, indole-H), $7.82(\mathrm{~s}, 1 \mathrm{H}$, benzothiazole-H), $7.64(\mathrm{~d}$, $J=8.0 \mathrm{~Hz}, 1 \mathrm{H}$, benzothiazole- $\mathrm{H}), 7.57-7.51(\mathrm{~m}, 1 \mathrm{H}$, benzothiazole- $\mathrm{H}), 7.37-7.30(\mathrm{~m}, 3 \mathrm{H}$, 3-indole-H), 7.30-7.19 (m, 5H, 5-benzyl-H), 5.48 (s, 2H, - $\left.\mathrm{CH}_{2^{-}}\right), 3.52$ (s, $\left.2 \mathrm{H},-\mathrm{CH}_{2^{-}}\right), 2.79$ (d, $J=$ $\left.8.8 \mathrm{~Hz}, 2 \mathrm{H},-\mathrm{CH}_{2}-\right), 2.01-1.82\left(\mathrm{~m}, 2 \mathrm{H},-\mathrm{CH}_{2}-\right), 1.56\left(\mathrm{~d}, J=11.6 \mathrm{~Hz}, 2 \mathrm{H},-\mathrm{CH}_{2}-\right), 1.41-1.24(\mathrm{~m}, 1 \mathrm{H}$, -CH-), 1.22-1.06 (m, 2H, - $\left.\mathrm{CH}_{2}-\right), 0.89\left(\mathrm{~d}, J=6.4 \mathrm{~Hz}, 3 \mathrm{H},-\mathrm{CH}_{3}\right) ;{ }^{13} \mathrm{C}$ NMR (100 MHz, DMSO- $\left.\left.d_{6}\right)\right)$ $\delta 159.44,152.57,148.25,141.13,137.98,137.31,134.05,133.72,132.08,129.10,128.03,127.61$, $127.49,125.36,123.28,122.19,121.90,121.30,119.76,111.30,111.20,62.80,53.70,49.85$, 34.35, 30.74, 22.28; Anal. Calcd, for $\mathrm{C}_{31} \mathrm{H}_{32} \mathrm{~N}_{6} \mathrm{OS}(\%): \mathrm{C}, 69.38 ; \mathrm{H}, 6.01 ; \mathrm{N}, 15.66$. Found (\%):C, 69.45; H, 6.09; N, 15.58 .

5.1.8.26.(E)-4-(6-((4-methylpiperidin-1-yl)methyl)benzothiazol-2-yl)-1-((1-(4-methylbenzyl)-1H-in dol-3-yl)methylene) semicarbazide (20r)

Yield: 48\%; M.p.: $187-189{ }^{\circ} \mathrm{C}$; MS (ESI) m/z: $551.4[\mathrm{M}+\mathrm{H}]^{+}$; IR $\left(\mathrm{KBr}, \mathrm{cm}^{-1}\right): 1616.3 ;{ }^{1} \mathrm{H}$ NMR (400 MHz, DMSO- $\left.\left.d_{6}\right)\right) \delta 11.07(\mathrm{~s}, 1 \mathrm{H},-\mathrm{NH}-), 10.41(\mathrm{~s}, 1 \mathrm{H},-\mathrm{NH}-), 8.26(\mathrm{~s}, 1 \mathrm{H},-\mathrm{CH}=)$, 8.25-8.22 (m, 1H, indole- $\mathrm{H}), 8.06(\mathrm{~s}, 1 \mathrm{H}$, indole- $\mathrm{H}), 7.84(\mathrm{~s}, 1 \mathrm{H}$, indole- $\mathrm{H}), 7.66(\mathrm{~d}, J=6.8 \mathrm{~Hz}$, $1 \mathrm{H}$, benzothiazole- $\mathrm{H}), 7.57-7.49(\mathrm{~m}, 1 \mathrm{H}$, benzothiazole- $\mathrm{H}), 7.35(\mathrm{~d}, J=6.8 \mathrm{~Hz}, 1 \mathrm{H}$, benzothiazole-H), 7.26-7.20 (m, 2H, 2-indole-H), 7.18 (d, $J=8.4 \mathrm{~Hz}, 2 \mathrm{H}, 2$-benzyl-H), 7.13 (d, $J$ $=8.4 \mathrm{~Hz}, 2 \mathrm{H}, 2$-benzyl-H), $5.42\left(\mathrm{~s}, 2 \mathrm{H},-\mathrm{CH}_{2^{-}}\right), 3.54\left(\mathrm{~s}, 2 \mathrm{H},-\mathrm{CH}_{2^{-}}\right), 2.97-2.70\left(\mathrm{~m}, 2 \mathrm{H},-\mathrm{CH}_{2^{-}}\right)$, 2.25 (s, 3H, - $\left.\mathrm{CH}_{3}\right), 2.07-1.78\left(\mathrm{~m}, 2 \mathrm{H},-\mathrm{CH}_{2}-\right), 1.66-1.49\left(\mathrm{~m}, 2 \mathrm{H},-\mathrm{CH}_{2}-\right), 1.44-1.27$ (m, $\left.1 \mathrm{H},-\mathrm{CH}-\right)$, 1.25-1.07 (m, $\left.2 \mathrm{H},-\mathrm{CH}_{2}-\right), 0.89\left(\mathrm{~d}, J=6.4 \mathrm{~Hz}, 3 \mathrm{H},-\mathrm{CH}_{3}\right) ;{ }^{13} \mathrm{C}$ NMR $\left(100 \mathrm{MHz}\right.$, DMSO- $\left.\left.d_{6}\right)\right) \delta$ $159.49,152.56,148.29,141.11,137.27,137.26,137.25,134.90,133.68,132.07,129.64,127.68$, $127.59,125.36,123.22,122.17,122.04,121.25,119.77,111.23,111.16,62.66,53.63,49.66$, 34.20, 30.65, 22.25, 21.12; Anal. Calcd, for $\mathrm{C}_{32} \mathrm{H}_{34} \mathrm{~N}_{6} \mathrm{OS}(\%): \mathrm{C}, 69.79 ; \mathrm{H}, 6.22 ; \mathrm{N}, 15.26$. Found (\%):C, 69.89; H, 6.32; N, 15.32 .

5.1.8.27.(E)-4-(6-((4-methylpiperidin-1-yl)methyl)benzothiazol-2-yl)-1-((1-(4-tert-butylbenzyl)-1H -indol-3-yl)methylene)semicarbazide (20s)

Yield: 48\%; M.p.: 206-208 ${ }^{\circ} \mathrm{C}$; MS (ESI) m/z: $593.5[\mathrm{M}+\mathrm{H}]^{+}$; IR $\left(\mathrm{KBr}, \mathrm{cm}^{-1}\right): 1617.2 ;{ }^{1} \mathrm{H}$ NMR (400 MHz, DMSO- $\left.\left.d_{6}\right)\right) \delta 11.11(\mathrm{~s}, 1 \mathrm{H},-\mathrm{NH}-), 10.41(\mathrm{~s}, 1 \mathrm{H},-\mathrm{NH}-), 8.27(\mathrm{~s}, 1 \mathrm{H},-\mathrm{CH}=)$, 8.27-8.23 (m, 1H, indole- $\mathrm{H}), 8.08(\mathrm{~s}, 1 \mathrm{H}$, indole- $\mathrm{H}), 7.93(\mathrm{~s}, 1 \mathrm{H}$, indole- $\mathrm{H}), 7.71(\mathrm{~d}, J=6.4 \mathrm{~Hz}$, $1 \mathrm{H}$, benzothiazole- $\mathrm{H}), 7.61-7.53(\mathrm{~m}, 1 \mathrm{H}$, benzothiazole- $\mathrm{H}), 7.42(\mathrm{~d}, J=7.2 \mathrm{~Hz}, 1 \mathrm{H}$, 
benzothiazole-H), 7.34 (d, $J=8.4 \mathrm{~Hz}, 2 \mathrm{H}, 2$-benzyl-H), 7.28-7.22 (m, 2H, indole- $\mathrm{H}), 7.20$ (d, $J=$ $8.4 \mathrm{~Hz}, 2 \mathrm{H}, 2$-benzyl-H), $5.43\left(\mathrm{~s}, 2 \mathrm{H},-\mathrm{CH}_{2^{-}}\right), 3.88\left(\mathrm{~s}, 2 \mathrm{H},-\mathrm{CH}_{2^{-}}\right), 3.19-2.92\left(\mathrm{~m}, 2 \mathrm{H},-\mathrm{CH}_{2^{-}}\right)$, 2.48-2.13 (m, $\left.2 \mathrm{H},-\mathrm{CH}_{2^{-}}\right), 1.74-1.55\left(\mathrm{~m}, 2 \mathrm{H},-\mathrm{CH}_{2^{-}}\right), 1.55-1.38(\mathrm{~m}, 1 \mathrm{H},-\mathrm{CH}-), 1.30-1.17(\mathrm{~m}, 11 \mathrm{H}$, 3- $\left.\mathrm{CH}_{3}\right), 0.90\left(\mathrm{~d}, J=6.4 \mathrm{~Hz}, 3 \mathrm{H},-\mathrm{CH}_{3}\right) ;{ }^{13} \mathrm{C}$ NMR $\left(150 \mathrm{MHz}, \mathrm{DMSO}-d_{6}\right) \delta 159.16,152.19,150.07$, $148.10,140.76,136.95,136.93,134.62,133.25,131.78,127.29$, 127.03, 125.47, 125.00, 122.91, 121.86, 121.76, 120.89, 119.51, 110.91, 110.83, 62.20, 53.18, 49.13, 34.29, 33.71, 31.15, 30.20, 21.85; Anal. Calcd, for $\mathrm{C}_{35} \mathrm{H}_{40} \mathrm{~N}_{6} \mathrm{OS}(\%): \mathrm{C}, 70.91 ; \mathrm{H}, 6.80 ; \mathrm{N}, 14.18$. Found (\%):C, 70.98; H, 6.75; $\mathrm{N}, 14.28$.

5.1.8.28.(E)-4-(6-((4-methylpiperidin-1-yl)methyl)benzothiazol-2-yl)-1-((1-(4-chlorobenzyl)-1H-in dol-3-yl)methylene) semicarbazide (20t)

Yield: 59\%; M.p.: 199-201 ${ }^{\circ} \mathrm{C}$; MS (ESI) m/z: $571.3[\mathrm{M}+\mathrm{H}]^{+}$; ${ }^{1} \mathrm{H}$ NMR $(600 \mathrm{MHz}$, DMSO- $\left.\left.d_{6}\right)\right) \delta 11.11(\mathrm{~s}, 1 \mathrm{H},-\mathrm{NH}-), 10.43(\mathrm{~s}, 1 \mathrm{H},-\mathrm{NH}-), 8.30-8.22(\mathrm{~m}, 2 \mathrm{H},-\mathrm{CH}=$, indole-H), 8.16-8.02 (m, 1H, indole- $\mathrm{H}), 7.90-7.78(\mathrm{~m}, 1 \mathrm{H}$, indole- $\mathrm{H}), 7.71-7.60(\mathrm{~m}, 1 \mathrm{H}$, benzothiazole- $\mathrm{H})$, 7.55-7.51 (m, 1H, benzothiazole-H), $7.40(\mathrm{~d}, J=8.4 \mathrm{~Hz}, 2 \mathrm{H}, 2$-benzyl-H), 7.38-7.32 (m, $1 \mathrm{H}$, benzothiazole-H), 7.28 (d, $J=8.4 \mathrm{~Hz}, 2 \mathrm{H}, 2$-benzyl-H), 7.26-7.22 (m, 2H, 2-indole-H), 5.48 (s, $\left.2 \mathrm{H},-\mathrm{CH}_{2}-\right), 3.53\left(\mathrm{~s}, 2 \mathrm{H},-\mathrm{CH}_{2^{-}}\right), 2.98-2.66\left(\mathrm{~m}, 2 \mathrm{H},-\mathrm{CH}_{2}\right), 2.17-1.75\left(\mathrm{~m}, 2 \mathrm{H},-\mathrm{CH}_{2^{-}}\right), 1.67-1.50$ $\left(\mathrm{m}, 2 \mathrm{H},-\mathrm{CH}_{2}-\right), 1.44-1.29(\mathrm{~m}, 1 \mathrm{H},-\mathrm{CH}-), 1.23-1.09\left(\mathrm{~m}, 2 \mathrm{H},-\mathrm{CH}_{2^{-}}\right), 0.89(\mathrm{~d}, J=6.4 \mathrm{~Hz}, 3 \mathrm{H}$, $-\mathrm{CH}_{3}$ ); Anal. Calcd, for $\mathrm{C}_{31} \mathrm{H}_{31} \mathrm{ClN}_{6} \mathrm{OS}(\%): \mathrm{C}, 65.19 ; \mathrm{H}, 5.47 ; \mathrm{N}, 14.71$. Found (\%):C, 65.26; H, $5.39 ; \mathrm{N}, 14.78$.

5.1.8.29.(E)-4-(6-((4-methylpiperidin-1-yl)methyl)benzothiazol-2-yl)-1-((1-(3-chlorobenzyl)-1H-in dol-3-yl)methylene)semicarbazide (20u)

Yield: 59\%; M.p.: 195-197 ${ }^{\circ} \mathrm{C}$; MS (ESI) m/z: $571.1[\mathrm{M}+\mathrm{H}]^{+}$; ${ }^{1} \mathrm{H}$ NMR $(600 \mathrm{MHz}$, DMSO- $\left.\left.d_{6}\right)\right) \delta 11.09$ (s, 1H, -NH-), 10.41 (s, 1H, -NH-), 8.31-8.24 (m, 2H, -CH=, indole-H), 8.17-8.05 (m, 1H, indole- $\mathrm{H}), 7.83(\mathrm{~s}, 1 \mathrm{H}$, benzothiazole- $\mathrm{H})$, 7.71-7.60 (m, 1H, benzothiazole- $\mathrm{H})$, 7.58-7.54 (m, 1H, benzothiazole- $\mathrm{H}), 7.39-7.30(\mathrm{~m}, 4 \mathrm{H}, 4$-benzyl- $\mathrm{H}), 7.27-7.20(\mathrm{~m}, 3 \mathrm{H}$, 3-indole- $\mathrm{H}), 5.50\left(\mathrm{~s}, 2 \mathrm{H},-\mathrm{CH}_{2^{-}}\right), 3.51\left(\mathrm{~s}, 2 \mathrm{H},-\mathrm{CH}_{2^{-}}\right), 2.88-2.67\left(\mathrm{~m}, 2 \mathrm{H},-\mathrm{CH}_{2^{-}}\right), 2.07-1.77(\mathrm{~m}, 2 \mathrm{H}$, $\left.-\mathrm{CH}_{2^{-}}\right), 1.65-1.47\left(\mathrm{~m}, 2 \mathrm{H},-\mathrm{CH}_{2^{-}}\right), 1.40-1.25(\mathrm{~m}, 1 \mathrm{H},-\mathrm{CH}-), 1.23-1.05\left(\mathrm{~m}, 2 \mathrm{H},-\mathrm{CH}_{2^{-}}\right), 0.89$ (d, J= $6.4 \mathrm{~Hz}, 3 \mathrm{H},-\mathrm{CH}_{3}$ ); Anal. Calcd, for $\mathrm{C}_{31} \mathrm{H}_{31} \mathrm{ClN}_{6} \mathrm{OS}(\%): \mathrm{C}, 65.19 ; \mathrm{H}, 5.47 ; \mathrm{N}, 14.71$. Found (\%): $\mathrm{C}$, 65.16; H, 5.49; N, 14.68.

5.1.8.30.(E)-4-(6-((4-methylpiperidin-1-yl)methyl)benzothiazol-2-yl)-1-((1-(2-chlorobenzyl)-1H-in dol-3-yl)methylene)semicarbazide (20v)

Yield: 59\%; M.p.: 208-211 ${ }^{\circ} \mathrm{C}$; MS (ESI) m/z: $571.3[\mathrm{M}+\mathrm{H}]^{+}$; ${ }^{1} \mathrm{H}$ NMR $(600 \mathrm{MHz}$, DMSO- $\left.\left.d_{6}\right)\right) \delta 11.10(\mathrm{~s}, 1 \mathrm{H},-\mathrm{NH}-), 10.43(\mathrm{~s}, 1 \mathrm{H},-\mathrm{NH}-), 8.33-8.28$ (m, 1H, indole-H), $8.27(\mathrm{~s}, 1 \mathrm{H}$, $-\mathrm{CH}=)$, 8.05-7.93 (m, 1H, indole- $\mathrm{H}), 7.83(\mathrm{~s}, 1 \mathrm{H}$, indole- $\mathrm{H})$, 7.69-7.60 $(\mathrm{m}, 1 \mathrm{H}$, benzothiazole- $\mathrm{H})$, $7.54(\mathrm{~d}, J=7.1 \mathrm{~Hz}, 1 \mathrm{H}$, benzothiazole-H), 7.50-7.45 (m, 1H, benzothiazole- $\mathrm{H}), 7.37-7.31(\mathrm{~m}, 2 \mathrm{H}$, indole-H), 7.29-7.23 (m, 3H, 3-benzyl-H), $6.81\left(\mathrm{~d}, J=7.5 \mathrm{~Hz}, 1 \mathrm{H}\right.$, benzyl-H), $5.58\left(\mathrm{~s}, 2 \mathrm{H},-\mathrm{CH}_{2}\right.$ ), $3.52\left(\mathrm{~s}, 2 \mathrm{H},-\mathrm{CH}_{2^{-}}\right), 2.87-2.73\left(\mathrm{~m}, 2 \mathrm{H},-\mathrm{CH}_{2^{-}}\right), 2.02-1.85\left(\mathrm{~m}, 2 \mathrm{H},-\mathrm{CH}_{2^{-}}\right), 1.62-1.53(\mathrm{~m}, 2 \mathrm{H}$, $\left.-\mathrm{CH}_{2^{-}}\right), 1.39-1.29(\mathrm{~m}, 1 \mathrm{H},-\mathrm{CH}-), 1.21-1.10\left(\mathrm{~m}, 2 \mathrm{H},-\mathrm{CH}_{2^{-}}\right), 0.89$ (d, J=6.4 Hz, 3H, - $\left.\mathrm{CH}_{3}\right)$; Anal. Calcd, for $\mathrm{C}_{31} \mathrm{H}_{31} \mathrm{ClN}_{6} \mathrm{OS}(\%): \mathrm{C}, 65.19 ; \mathrm{H}, 5.47 ; \mathrm{N}, 14.71$. Found (\%):C, 65.33; H, 5.45; N, 14.61 . 5.1.8.31.(E)-4-(6-((4-methylpiperidin-1-yl)methyl)benzothiazol-2-yl)-1-((1-(2,4-dichlorobenzyl)-1 H-indol-3-yl)methylene)semicarbazide (20w)

Yield: 59\%; M.p.: $195-197{ }^{\circ} \mathrm{C}$; MS (ESI) m/z: $605.1[\mathrm{M}+\mathrm{H}]^{+}$; ${ }^{1} \mathrm{H}$ NMR $(400 \mathrm{MHz}$, DMSO- $\left.\left.d_{6}\right)\right) \delta 11.09$ (s, $\left.1 \mathrm{H},-\mathrm{NH}-\right), 10.46(\mathrm{~s}, 1 \mathrm{H},-\mathrm{NH}-), 8.36-8.28$ (m, 1H, indole-H), $8.27(\mathrm{~s}, 1 \mathrm{H}$, $-\mathrm{CH}=), 7.98(\mathrm{~s}, 1 \mathrm{H}$, indole-H), $7.83(\mathrm{~s}, 1 \mathrm{H}$, indole- $\mathrm{H}), 7.72(\mathrm{~d}, J=1.6 \mathrm{~Hz}, 1 \mathrm{H}$, benzothiazole-H), 
$7.65(\mathrm{~d}, J=7.6 \mathrm{~Hz}, 1 \mathrm{H}$, benzothiazole- $\mathrm{H}), 7.52-7.43(\mathrm{~m}, 1 \mathrm{H}$, benzothiazole- $\mathrm{H}), 7.40-7.31(\mathrm{~m}, 2 \mathrm{H}$, 2-indole-H), 7.30-7.20 (m, 2H, 2-benzyl-H), 6.80 (d, $J=7.6 \mathrm{~Hz}, 1 \mathrm{H}$, benzyl-H), $5.56(\mathrm{~s}, 2 \mathrm{H}$, $\left.-\mathrm{CH}_{2^{-}}\right), 3.57\left(\mathrm{~s}, 2 \mathrm{H},-\mathrm{CH}_{2^{-}}\right), 2.93-2.73\left(\mathrm{~m}, 2 \mathrm{H},-\mathrm{CH}_{2^{-}}\right), 2.19-1.80\left(\mathrm{~m}, 2 \mathrm{H},-\mathrm{CH}_{2^{-}}\right), 1.57(\mathrm{~d}, J=11.6$ $\left.\mathrm{Hz}, 2 \mathrm{H},-\mathrm{CH}_{2}-\right), 1.43-1.26(\mathrm{~m}, 1 \mathrm{H},-\mathrm{CH}-), 1.25-1.09\left(\mathrm{~m}, 2 \mathrm{H},-\mathrm{CH}_{2^{-}}\right), 0.88(\mathrm{~d}, J=6.4 \mathrm{~Hz}, 3 \mathrm{H}$, $-\mathrm{CH}_{3}$ ); Anal. Calcd, for $\mathrm{C}_{31} \mathrm{H}_{30} \mathrm{Cl}_{2} \mathrm{~N}_{6} \mathrm{OS}(\%): \mathrm{C}, 61.48 ; \mathrm{H}, 4.99 ; \mathrm{N}, 13.88$. Found (\%):C, 61.56; H, $4.88 ; \mathrm{N}, 13.92$.

5.2. Pharmacology and the procedure for QSAR modeling (see supplementary information)

\section{Acknowledgments}

The work was supported by Program for Liaoning Excellent Talents in University (LR2014030), National Natural Science Foundation of China (81102470) and National High Technology Research and Development Program of China (863 Program) (2012AA020305).

\section{References}

[1] http://www.who.int/mediacentre/factsheets/fs297/en/ (WHO Media Centre Cancer Feb 8, last accessed: 26.03.12).

[2] D. Zhou, P. Zhou, D. A. Evrard, K. Meagher, M. Webb, B. L. Harrison, D. M. Huryn, J. Golembieski, G. A. Hornby, L. E. Schechter, D. L. Smith, T. H. Andree, R. E. Mewshaw, Studies toward the discovery of the next generation of antidepressants. Part 6: Dual 5-HT1A receptor and serotonin transporter affinity within a class of arylpiperazinyl-cyclohexyl indole derivatives, Bioorg. Med. Chem. 16 (2008) 6707-6723.

[3] P. Ahuja, N. Siddiqui, Anticonvulsant evaluation of clubbed indole-1,2,4-triazine derivatives: A synthetic approach, Eur. J. Med. Chem, 80 (2014) 509-522.

[4] M.Z. Zhang, N. Mulholland, D. Beattie, D. Irwin, Y.C. Gu, Q. Chen, G.F. Yang, J. Clough, Synthesis and antifungal activity of 3-(1,3,4-oxadiazol-5-yl)-indoles and 3-(1,3,4-oxadiazol-5-yl)methyl-indoles, Eur. J. Med. Chem. 63 (2013) 22-32.

[5] M. Z. Zhang, Q. Chen, G. F. Yang, A review on recent developments of indole-containing antiviral agents, Eur. J. Med. Chem. 89 (2015) 421-441.

[6] M. A. A. Radwan, E. A. Ragab, N. M. Sabry, S. M. ElShenawy, Synthesis and biological evaluation of new 3-substituted indole derivatives as potential anti-inflammatory and analgesic agents, Bioorg. Med. Chem. 15 (2007) 3832-3841.

[7] R. Álvarez, P. Puebla, J. F. Díaz, A. C. Bento, R. GarcíaNavas, J. de la IglesiaVicente, F. Mollinedo, J. M. Andreu, M. Medarde, R. Peláez, Article Previous Article Next Article Table of Contents Endowing Indole-Based Tubulin Inhibitors with an Anchor for Derivatization: Highly Potent 3-Substituted Indolephenstatins and Indoleisocombretastatins, J. Med. Chem. 56 (2013) 2813-2827.

[8] S. Oishi, T. Watanabe, J. I. Sawada, A. Asai, H. Ohno, N. Fujii, Endowing Indole-Based Tubulin Inhibitors with an Anchor for Derivatization: Highly Potent 3-Substituted Indolephenstatins and Indoleisocombretastatins, J. Med. Chem. 53 (2010) 5054-5058.

[9] A. Andreani, S. Burnelli, M. Granaiola, A. Leoni, A. Locatelli, R. Morigi, M. Rambaldi, L. Varoli, L. Landi, C. Prata, M. V. Berridge, C. Grasso, H. H. Fiebig, G. Kelter, A. M. Burger, M. W. Kunkel, Antitumor Activity of Bis-indole Derivatives, J. Med. Chem. 51 (2008) 4563-4570.

[10] J. L. Hyatt, T. Moak, M. J. Hatfield, L. Tsurkan, C. C. Edwards, M. Wierdl, M. K. Danks, R. M. Wadkins, P. M. Potter, Selective Inhibition of Carboxylesterases by Isatins, 
Indole-2,3-diones, J. Med. Chem. 50 (2007) 1876-1885.

[11] M. Nguyen, R. C. Marcellus, A. Roulston, M. Watson, L. Serfass, S. R. Murthy Madiraju, D. Goulet, J. Viallet, L. Bélec, X. Billot, S. Acoca, E. Purisima, A. Wiegmans, L. Cluse, R. W. Johnstone, P. Beauparlant, G. C. Shore, Small molecule obatoclax (GX15-070) antagonizes MCL-1 and overcomes MCL-1-mediated resistance to apoptosis, Proc Natl Acad Sci USA. 104 (2007) 19512-19517.

[12] L. Sun, C. Liang, S. Shirazian, Y. Zhou, T. Miller, J. Cui, J. Y. Fukuda, J.Y. Chu, A. Nematalla, X. Wang, H. Chen, A. Sistla, T. C. Luu, F. Tang, J. Wei, C. Tang, Discovery of 5-[5-Fluoro-2-oxo-1,2-dihydroindol-(3Z)-ylidenemethyl]-2,4-dimethyl-1H-pyrrole-3-carboxy lic Acid (2-Diethylaminoethyl)amide, a Novel Tyrosine Kinase Inhibitor Targeting Vascular Endothelial and Platelet-Derived Growth Factor Receptor Tyrosine Kinase, J. Med. Chem. 46 (2003) 1116-1119.

[13] S. Wu, L. Wang, W. Guo, X. Liu, J. Liu, X. Wei, B. Fang, Analogues and Derivatives of Oncrasin-1, a Novel Inhibitor of the C-Terminal Domain of RNA Polymerase II and Their Antitumor Activities, J. Med. Chem. 54 (2011) 2668-2679.

[14] G. Bacher, B. Nickel, P. Emig, U. Vanhoefer, S. Seeber, A. Shandra, T. Klenner, T. Beckers, D-24851, a Novel Synthetic Microtubule Inhibitor, Exerts Curative Antitumoral Activity in Vivo, Shows Efficacy toward Multidrug-resistant Tumor Cells, and Lacks Neurotoxicity, Cancer Res. 61 (2001) 392-399.

[15] W. Guo, S. Wu, L. Wang, R.Y. Wang, X. Wei, J. Liu, B. Fang, Interruption of RNA processing machinery by a small compound, 1-[(4-chlorophenyl)methyl]-1H-indole-3carboxaldehyde(oncrasin-1), Mol. Cancer Ther. 8 (2009) 441-448.

[16] J. Das, R. V. Moquin, J. Lin, C. Liu, A. M. Doweyko, H. F. DeFex, Q. Fang, S. Pang, S. Pitt, D. R. Shen, Discovery of 2-amino-heteroaryl-benzothiazole-6-anilides as potent $\mathrm{p} 56^{\text {lck }}$ inhibitors, Bioorg. Med. Chem. Lett. 13 (2003) 2587-2590.

[17] K. Yamazaki, Y. Kaneko, K. Suwa, S. Ebara, K. Nakazawa, K. Yasuno, Synthesis of potent and selective inhibitors of Candida albicans $N$-myristoyltransferase based on the benzothiazole structure, Bioorg. Med. Chem. 13 (2005) 2509-2522.

[18] S. Saeed, N. Rashid, P. G. Jones, M. Ali, R. Hussain, Synthesis, characterization and biological evaluation of some thiourea derivatives bearing benzothiazole moiety as potential antimicrobial and anticancer agents, Eur. J. Med. Chem. 45 (2010) 1323-1331.

[19] E. Kashiyama, I. Hutchinson, M. S. Chua, S. F. Stinson, L. R. Phillips, G. Kaur, E. A. Sausville, T. D. Bradshaw, A. D. Westwell, M. F. Stevens, Antitumor Benzothiazoles. 8. Synthesis, Metabolic Formation, and Biological Properties of the C-and N-Oxidation Products of Antitumor 2-(4-Aminophenyl)benzothiazoles, J. Med. Chem. 42 (1999) 4172-4184.

[20] A. Kamal, J. R. Tamboli, V. L. Nayak, S. Adil, M. Vishnuvardhan, S. Ramakrishna, Synthesis of pyrazolo[1,5-a]pyrimidine linked aminobenzothiazole conjugates as potential anticancer agents, Bioorg. Med. Chem. Lett. 23 (2013) 3208-3215.

[21] J. Ma, D. Chen, K. Lu, L. Wang, X. Han, Y. Zhao, P. Gong, Design, synthesis, and structure-activity relationships of novel benzothiazole derivatives bearing the ortho-hydroxy N-carbamoylhydrazone moiety as potent antitumor agents, Eur. J. Med. Chem. 86 (2014) 257-269.

[22] M. Qin, X. Zhai, H. Xie, J. Ma, K. Lu, Y. Wang, L. Wang, Y. Gu, P. Gong, Design and 
synthesis of novel 2-(4-(2-(dimethylamino)ethyl)-4H-1,2,4-triazol-3-yl)pyridines as potential antitumor agents, Eur. J. Med. Chem. 81 (2014) 47-58.

[23] Z. Liu, S. Wu, Y. Wang, R. Li, J. Wang, L. Wang, Y. Zhao, P. Gong, Design, synthesis and biological evaluation of novel thieno[3,2-d]pyrimidine derivatives possessing diaryl semicarbazone scaffolds as potent antitumor agents, Eur. J. Med. Chem. 87 (2014) 782-793.

[24] B. Qi, B. Mi, X. Zhai, Z. Xu, X. Zhang, Z. Tian, P. Gong, Discovery and optimization of novel 4-phenoxy-6,7-disubstituted quinolines possessing semicarbazones as c-Met kinase inhibitors, Bioorg. Med. Chem. 21 (2013) 5246-5260.

[25] R. V. Patel, P. K. Patel, P. Kumari, D. P. Rajani, K. H. Chikhalia, Synthesis of benzimidazolyl-1,3,4-oxadiazol-2ylthio-N-phenyl (benzothiazolyl) acetamides as antibacterial, antifungal and antituberculosis agents, Eur. J. Med. Chem. 53 (2012) 41-51.

[26] N. Kaila, K. Janz, A. Huang, A. Moretto, S. DeBernardo, P. W. Bedard, S. Tam, V. Clerin, J. C. Keith, D. H. H. Tsao, N. Sushkova, G. D. Shaw, R. T. Camphausen, R. G. Schaub, Q. Wang, 2-(4-chlorobenzyl)-3-hydroxy-7,8,9,10-tetrahydrobenzo[H]quinoline-4-carboxylic Acid (PSI-697): Identification of a Clinical Candidate from the Quinoline Salicylic Acid Series of P-Selectin Antagonists, J. Med. Chem. 50 (2006) 40-64.

[27] R. Thirumurugan, D. Sriram, A. Saxena, J. Stables, P. Yogeeswari, 2,4-Dimethoxyphenylsemicarbazones with anticonvulsant activity against three animal models of seizures: Synthesis and pharmacological evaluation, Bioorg. Med. Chem. 14 (2006) 3106-3112.

[28] M. Ando, K. Yoshikawa, Y. Iwase, S. Ishiura, Usefulness of Monitoring $\gamma-\mathrm{H} 2 \mathrm{AX}$ and Cell Cycle Arrest in HepG2 Cells for Estimating Genotoxicity Using a High-Content Analysis System, J. Biomol. Screen. 19 (2014) 1246-1254.

[29] K. S. Putt, G. W. Chen, J. M. Pearson, J. S. Sandhorst, M. S. Hoagland, J.T. Kwon, S. K. Hwang, H. Jin, M. I. Churchwell, M. H. Cho, Small-molecule activation of procaspase-3 to caspase-3 as a personalized anticancer strategy, Nat. Chem. Biol. 2 (2006) 543-550.

\section{Legends}

Fig. 1. Structures of compounds bearing indole-based moiety

Fig. 2. Structures of benzothiazole and semicarbazone derivatives

Fig. 3. The design of target compounds

Scheme 1. Reagents and conditions: (i) acetonitrile, amine, rt, 3 h; (ii) $80 \%$ hydrazine monohydrate, $\mathrm{FeCl}_{3} \cdot 6 \cdot \mathrm{H}_{2} \mathrm{O}$, activated carbon, ethanol, $65-78{ }^{\circ} \mathrm{C}$, 5 hours; (iii) $\mathrm{Br}_{2}, \mathrm{NH}_{4} \mathrm{SCN}$, acetic acid, $10{ }^{\circ} \mathrm{C}$ to rt, 4-6 hours; (iv) phenyl chloroformate, pyridine, $\mathrm{CH}_{2} \mathrm{Cl}_{2}, 0{ }^{\circ} \mathrm{C}$ to rt, 4-6 hours; (v) $80 \%$ hydrazine monohydrate, 1,4 -dioxane, $80^{\circ} \mathrm{C}, 6$ hours.

Scheme 2. Reagents and conditions: (i) substituted benzyl chloride, $\mathrm{K}_{2} \mathrm{CO}_{3}$, DMF, $80{ }^{\circ} \mathrm{C}, 3$ hours; (ii) $\mathrm{Na}_{2} \mathrm{SO}_{4}, \mathrm{NH}_{2} \mathrm{OH}, \mathrm{CCl}_{3} \mathrm{CH}(\mathrm{OH})_{2}, 95{ }^{\circ} \mathrm{C}$; (iii) conc. $\mathrm{H}_{2} \mathrm{SO}_{4}, 50-75{ }^{\circ} \mathrm{C}, 80{ }^{\circ} \mathrm{C}, 30$ minute; (iv) acetic acid, ethanol, reflux.

Fig. 4. NOE of the representative compound 20a

Table 1. Antitumor activity of target compounds 18a-c, 19a-e and 20a-w against HT-29, H460, A549 and MDA-MB-231 cancer cell lines.

Table 2. Enzymatic activity of the selected kinases by compounds 19a, 20d and 20e.

Fig.5 HCAS-mediated cell-cycle analysis of HT29 cancer cells, which were treated with either DMSO or compounds $20 \mathrm{~d}$ at $10 \mu \mathrm{M}$ for 48 hours. The graph showed the percentage of cells in each phase. 
Fig.6. (A) Structure of the template compound 20d, common substructure is in bold, (B) 3D structure superimposition of target compounds in the training set using compound 20d as the template.

Fig. 7. The plot of the predicted data versus the observed $\mathrm{pIC}_{50}$ of target compounds of training set and test set.

Fig.8. (A) 3D-QSAR model coefficients on van der Waals grids. Steric map (A) indicates areas where steric bulk is predicted to increase (green) or decrease (yellow) activity. (B) 3D-QSAR model coefficients on electrostatic potential grids. Electrostatic map indicates red contours around regions where high electron density (negative charge) is expected to increase activity, and blue contours represent areas where low electron density (partial positive charge) is expected to increase activity. 
Table 1. Antitumor activity of target compounds 18a-c, 19a-e and 20a-w against HT-29, H460, A549 and MDA-MB-231 cancer cell lines.

\begin{tabular}{|c|c|c|c|c|c|c|}
\hline & $a-c$ & & 19 & & & \\
\hline \multirow{2}{*}{ Compd. } & \multirow{2}{*}{$\mathrm{R}^{1}$} & \multirow{2}{*}{$\mathrm{R}$} & \multicolumn{4}{|c|}{$\mathrm{IC}_{50}(\mu \mathrm{mol} / \mathrm{L})$} \\
\hline & & & HT29 & H460 & A549 & MDA-MB-231 \\
\hline $18 \mathrm{a}$ & & - & $9.9 \pm 1.21$ & $2.55 \pm 0.28$ & $3.3 \pm 0.41$ & $1.19 \pm 0.09$ \\
\hline $18 \mathrm{~b}$ & & - & $12.1 \pm 1.33$ & $3.14 \pm 0.71$ & $3.3 \pm 0.51$ & $1.9 \pm 0.11$ \\
\hline $18 \mathrm{c}$ & & - & $15.2 \pm 2.01$ & $4.13 \pm 0.19$ & $3.58 \pm 0.21$ & $2.46 \pm 0.16$ \\
\hline $19 \mathrm{a}$ & & $5-\mathrm{F}$ & $0.97 \pm 0.10$ & $1.55 \pm 0.09$ & $2.31 \pm 0.18$ & ND \\
\hline $19 \mathrm{~b}$ & & $5-\mathrm{Cl}$ & $2.57 \pm 0.13$ & $1.17 \pm 0.16$ & $3.18 \pm 0.09$ & ND \\
\hline $19 \mathrm{c}$ & & $5-\mathrm{F}$ & $1.55 \pm 0.31$ & $1.06 \pm 0.14$ & $2.01 \pm 0.21$ & ND \\
\hline $19 \mathrm{~d}$ & & $5-\mathrm{OCF}_{3}$ & $1.90 \pm 0.11$ & $2.11 \pm 0.17$ & $1.99 \pm 0.34$ & ND \\
\hline $19 \mathrm{e}$ & & $5-\mathrm{F}$ & $4.10 \pm 0.33$ & $1.43 \pm 0.28$ & $3.01 \pm 0.15$ & ND \\
\hline $20 \mathrm{a}$ & & $\mathrm{H}$ & $0.041 \pm 0.007$ & $0.40 \pm 0.04$ & $2.9 \pm 0.12$ & $1.34 \pm 0.21$ \\
\hline $20 \mathrm{~b}$ & & $4-\mathrm{CH}_{3}$ & $0.036 \pm 0.005$ & $0.20 \pm 0.05$ & $1.6 \pm 0.09$ & $1.22 \pm 0.12$ \\
\hline $20 \mathrm{c}$ & & $4-\mathrm{C}\left(\mathrm{CH}_{3}\right)_{3}$ & $0.18 \pm 0.05$ & $0.81 \pm 0.10$ & $1.85 \pm 0.11$ & $2.23 \pm 0.16$ \\
\hline $20 \mathrm{~d}$ & & $4-\mathrm{Cl}$ & $0.024 \pm 0.004$ & $0.29 \pm 0.03$ & $0.84 \pm 0.08$ & $0.88 \pm 0.02$ \\
\hline $20 \mathrm{e}$ & & $3-\mathrm{Cl}$ & $0.040 \pm 0.01$ & $0.42 \pm 0.03$ & $1.24 \pm 0.14$ & $1.14 \pm 0.07$ \\
\hline $20 f$ & & $2-\mathrm{Cl}$ & $0.10 \pm 0.02$ & $1.6 \pm 0.11$ & $1.08 \pm 0.06$ & $1.18 \pm 0.03$ \\
\hline $20 \mathrm{~g}$ & & $3,4-(\mathrm{Cl})_{2}$ & $0.26 \pm 0.04$ & $1.36 \pm 0.08$ & $0.94 \pm 0.05$ & $1.0 \pm 0.11$ \\
\hline $20 \mathrm{~h}$ & & $2,4-(\mathrm{Cl})_{2}$ & $0.58 \pm 0.04$ & $0.95 \pm 0.03$ & $2.00 \pm 0.08$ & $1.41 \pm 0.06$ \\
\hline $20 \mathrm{i}$ & & $\mathrm{H}$ & $0.069 \pm 0.011$ & $0.45 \pm 0.02$ & $3.33 \pm 0.11$ & $1.47 \pm 0.09$ \\
\hline $20 \mathrm{j}$ & & $4-\mathrm{CH}_{3}$ & $0.058 \pm 0.009$ & $0.32 \pm 0.01$ & $1.51 \pm 0.25$ & 2. $01 \pm 0.17$ \\
\hline $20 \mathrm{k}$ & & 4- $\mathrm{C}\left(\mathrm{CH}_{3}\right)_{3}$ & $0.66 \pm 0.02$ & $1.67 \pm 0.12$ & $1.71 \pm 0.11$ & $2.12 \pm 0.23$ \\
\hline 201 & & $4-\mathrm{Cl}$ & $0.075 \pm 0.008$ & $0.37 \pm 0.03$ & $2.94 \pm 0.18$ & $2.57 \pm 0.22$ \\
\hline $20 \mathrm{~m}$ & & $3-\mathrm{Cl}$ & $0.081 \pm 0.011$ & $0.22 \pm 0.03$ & $2.41 \pm 0.09$ & $2.05 \pm 0.12$ \\
\hline $20 n$ & & $2-\mathrm{Cl}$ & $0.46 \pm 0.04$ & $0.98 \pm 0.05$ & $1.39 \pm 0.16$ & $0.90 \pm 0.04$ \\
\hline 200 & & $3,4-(\mathrm{Cl})_{2}$ & $0.63 \pm 0.02$ & $0.61 \pm 0.04$ & $1.47 \pm 0.14$ & $4.49 \pm 0.21$ \\
\hline $20 p$ & & $2,4-(\mathrm{Cl})_{2}$ & $1.04 \pm 0.05$ & $1.08 \pm 0.02$ & $1.90 \pm 0.07$ & $3.28 \pm 0.15$ \\
\hline $20 q$ & & $\mathrm{H}$ & $2.05 \pm 0.08$ & $1.91 \pm 0.18$ & $2.34 \pm 0.15$ & $2.85 \pm 0.25$ \\
\hline $20 \mathrm{r}$ & & 4- $\mathrm{CH}_{3}$ & $1.03 \pm 0.07$ & $0.41 \pm 0.05$ & $2.18 \pm 0.15$ & $1.14 \pm 0.13$ \\
\hline $20 \mathrm{~s}$ & & $4-\mathrm{C}\left(\mathrm{CH}_{3}\right)_{3}$ & $1.50 \pm 0.11$ & $3.37 \pm 0.12$ & $3.71 \pm 0.22$ & $3.88 \pm 0.37$ \\
\hline $20 t$ & & $4-\mathrm{Cl}$ & $0.75 \pm 0.06$ & $0.40 \pm 0.08$ & $2.03 \pm 0.15$ & $2.85 \pm 0.14$ \\
\hline $20 u$ & & $3-\mathrm{Cl}$ & $0.73 \pm 0.03$ & $0.28 \pm 0.07$ & $2.81 \pm 0.16$ & $2.11 \pm 0.13$ \\
\hline $20 v$ & & $2-\mathrm{Cl}$ & $1.57 \pm 0.13$ & $1.08 \pm 0.13$ & $3.33 \pm 0.14$ & $1.36 \pm 0.07$ \\
\hline $20 w$ & & $2,4-(\mathrm{Cl})_{2}$ & $2.12 \pm 0.18$ & $1.66 \pm 0.23$ & $3.64 \pm 0.21$ & $3.14 \pm 0.14$ \\
\hline PAC $-1^{\mathrm{a}}$ & - & - & $1.02 \pm 0.12$ & $1.25 \pm 0.09$ & $2.51 \pm 0.15$ & $3.2 \pm 0.12$ \\
\hline oncrasin- $1^{\text {a }}$ & - & - & $1.71 \pm 0.22$ & $0.85 \pm 0.03$ & $2.48 \pm 0.36$ & $26.1 \pm 1.01$ \\
\hline
\end{tabular}

ND: Not determined.

${ }^{\mathrm{a}}$ Used as the positive control. 
Table 2. Enzymatic activity of 8 selected kinases by compounds $19 \mathrm{a}, 20 \mathrm{~d}$ and $20 \mathrm{e}$.

\begin{tabular}{|c|c|c|c|c|c|c|c|c|}
\hline \multirow[t]{2}{*}{ Compd. } & \multicolumn{2}{|c|}{$\%$ Activation at $10 \mu \mathrm{M}$} & \multicolumn{5}{|c|}{$\%$ Inhibition at $10 \mu \mathrm{M}$} & \multirow[b]{2}{*}{ KIT } \\
\hline & Procapase-3 & c-met & Tubulin & KDR & $\mathrm{PDGFR}_{\beta}$ & FLT3 & EGFR & \\
\hline $19 a$ & 9.1 & 12.5 & - & 3.7 & 2.2 & 34.1 & 11.2 & 28.8 \\
\hline 20d & 70.9 & 20.4 & 26 & 5.9 & 12.8 & 4.1 & 19.6 & 2.0 \\
\hline $20 \mathrm{e}$ & 48.8 & 10.6 & 18 & - & - & - & - & - \\
\hline Positive control & PAC-1 & Foretinib & Cochicine & - & - & - & - & - \\
\hline
\end{tabular}

${ }^{\text {a }}$ Data presented is the mean value of two independent determinations. 


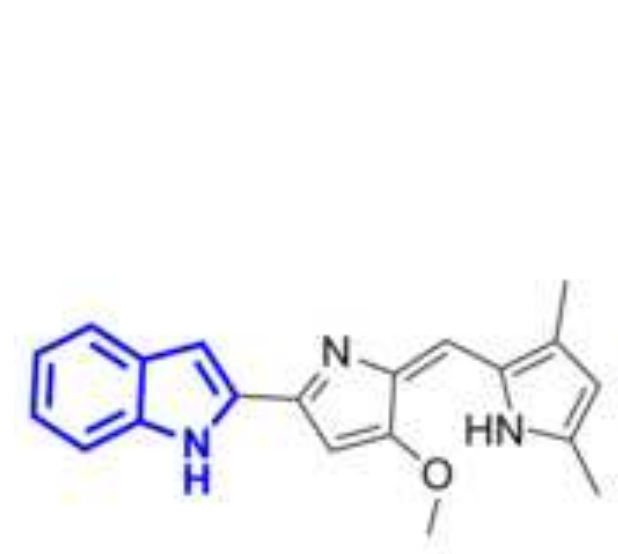

1 (Obatoclax, GX15-070, Phase III)

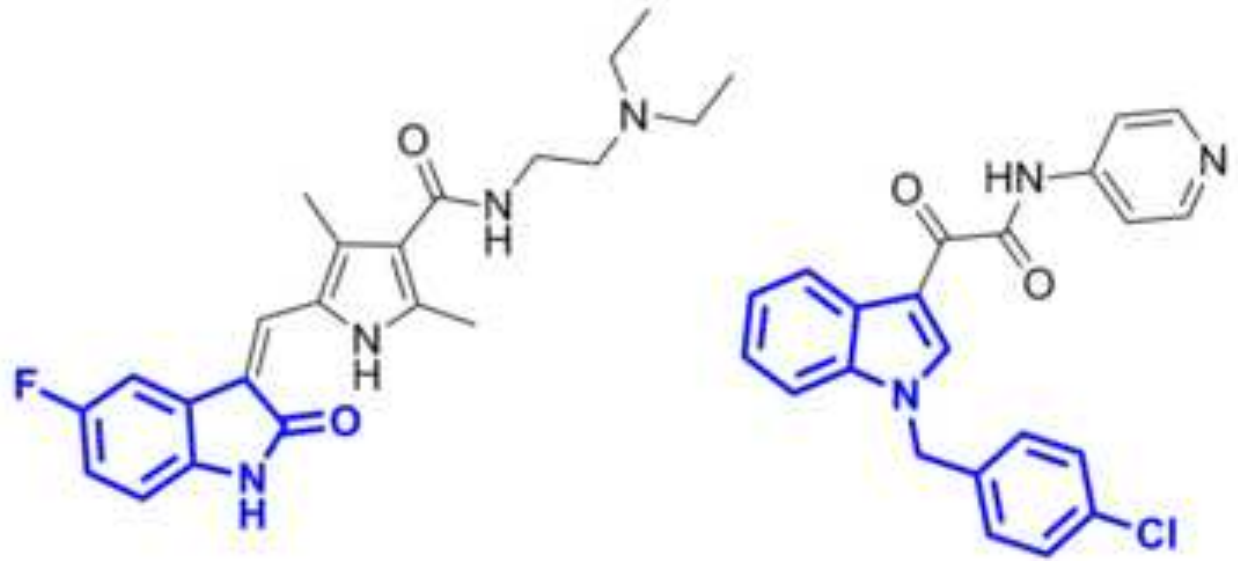

3 (Indibulin, D-24581, Phase I/II)

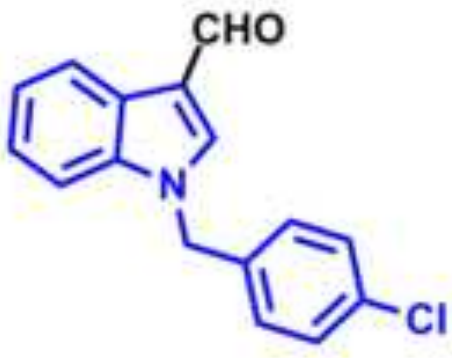

4 (oncrasin-1) 


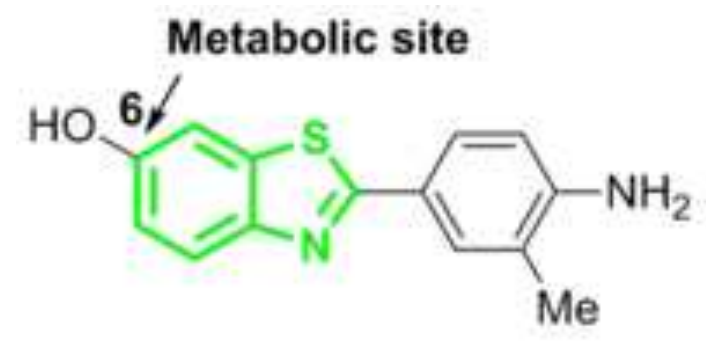

5 (NSC 703785)

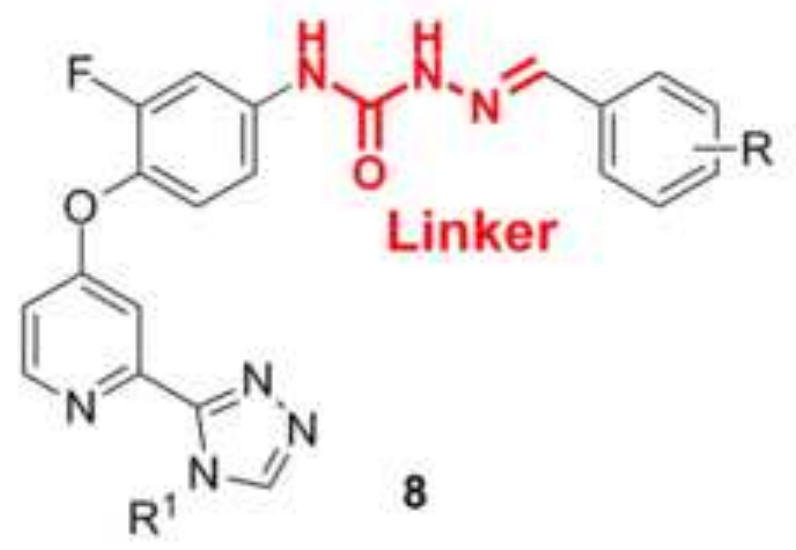

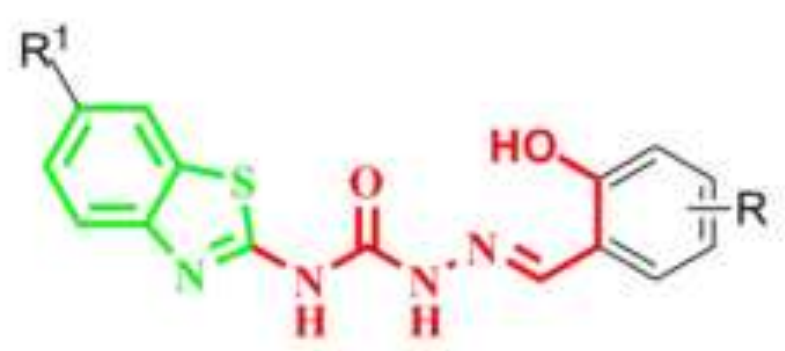

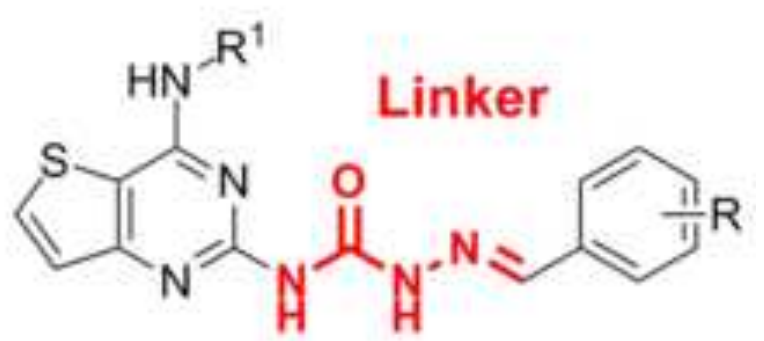

6

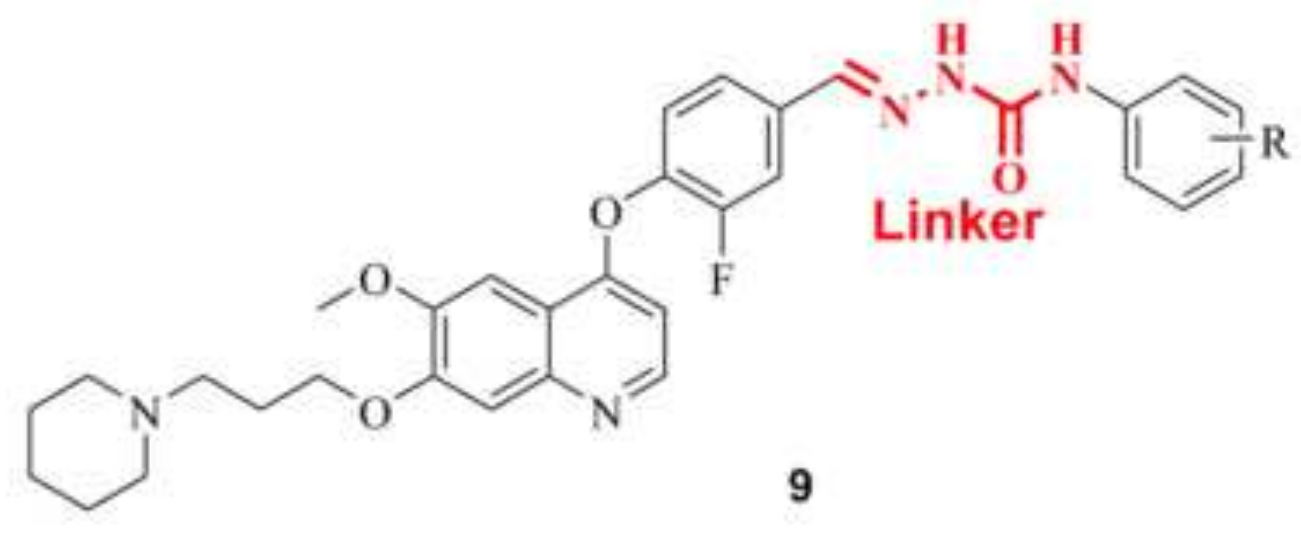



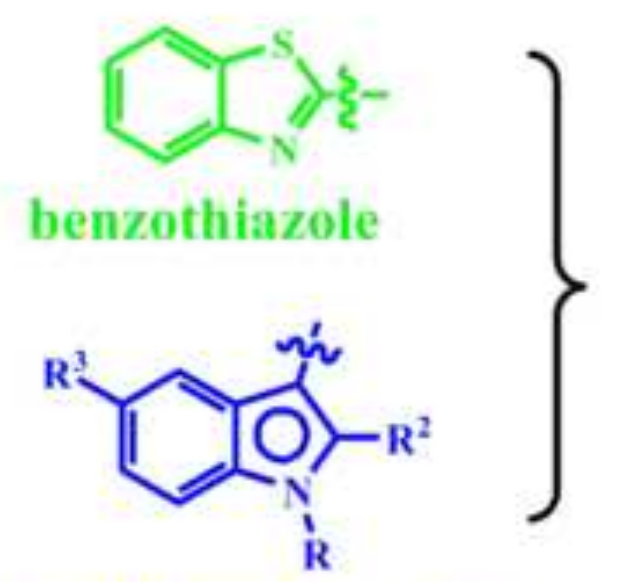

aliphatic amines

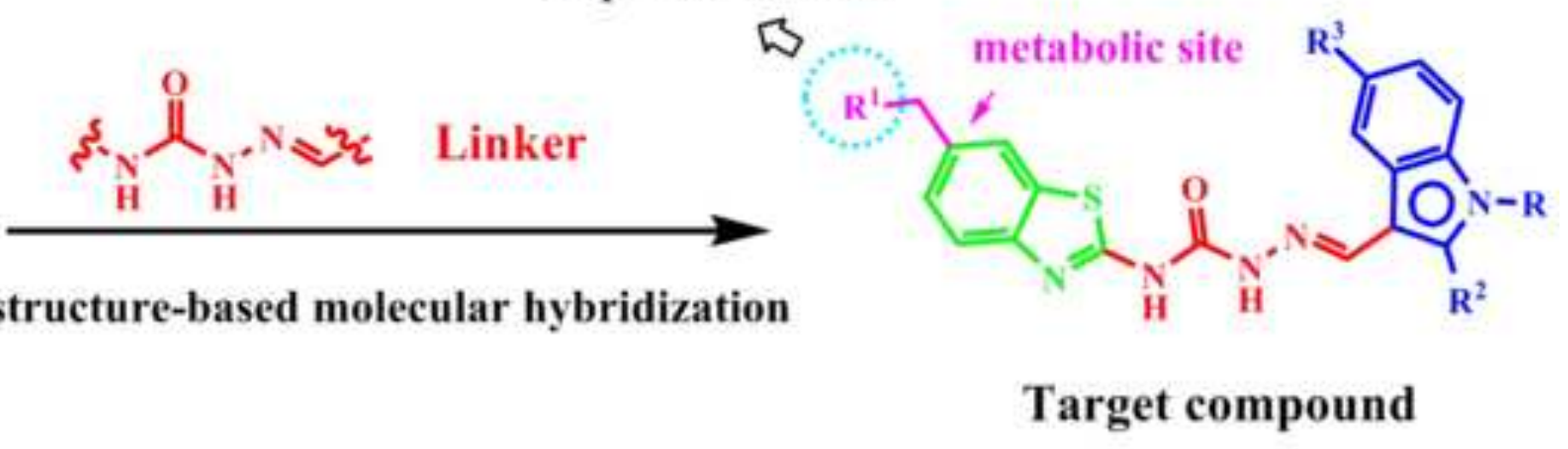

indole-based moiety 

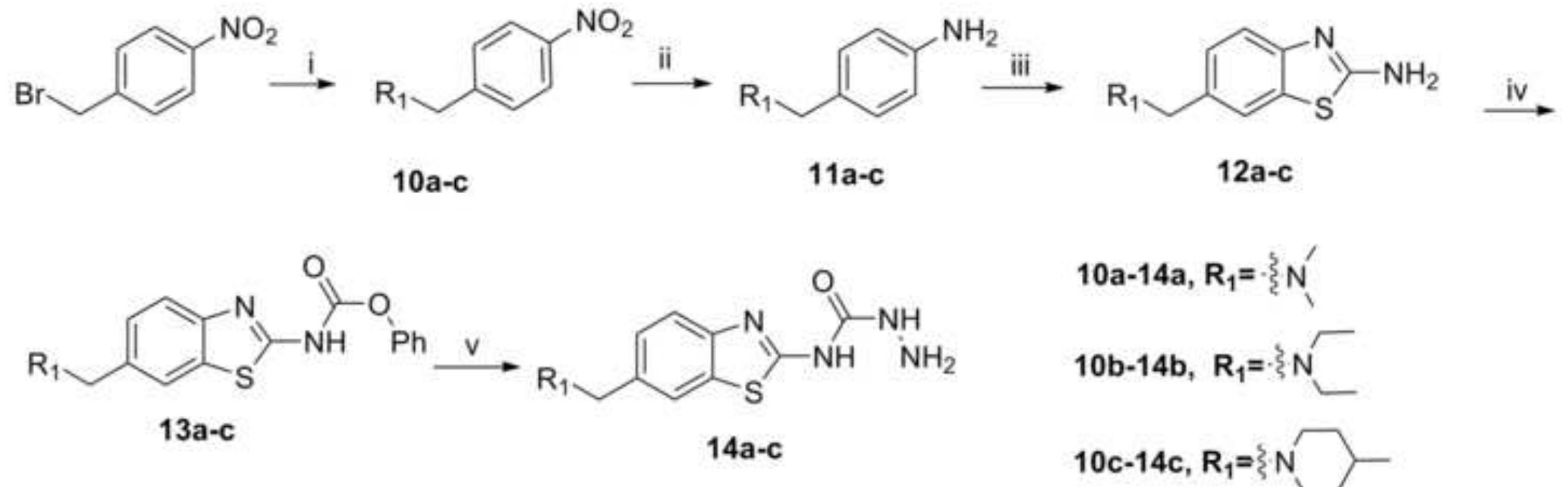

10a-14a, $R_{1}=-\xi N^{\prime}$

10b-14b, $R_{1}=-\xi$

10c-14c, $R_{1}=\xi N$

$13 a-c$

$14 a-c$

$10 c-14 c, R_{1}=\xi N$

$\overbrace{10 \mathrm{a}-\mathrm{c}}^{\mathrm{NO}} \stackrel{\mathrm{N}}{\longrightarrow}$

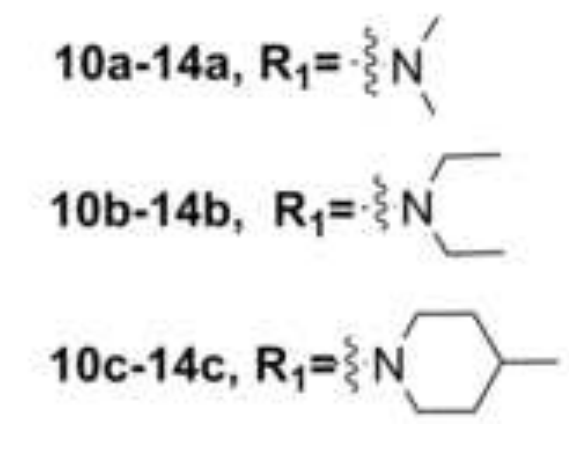

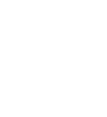




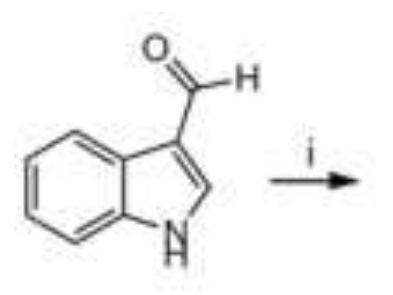

15

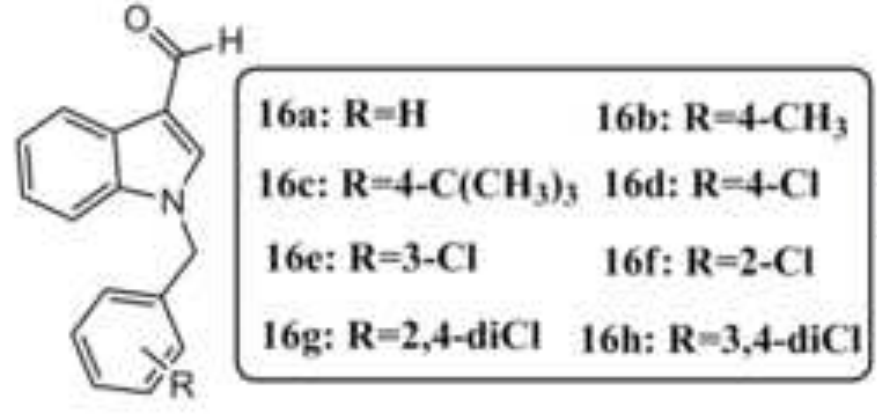

16a-h

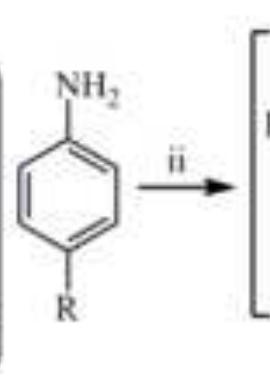

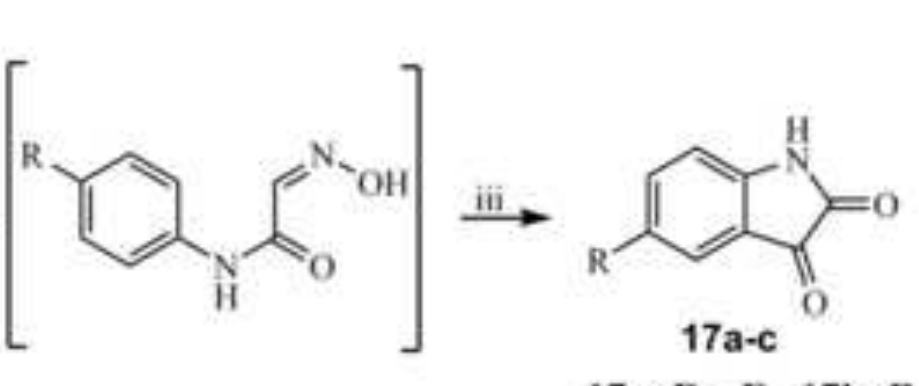

17a: $\mathrm{R}=\mathrm{F} \quad 17 \mathrm{~b}: \mathrm{R}=\mathrm{Cl}$

$17 \mathrm{e}: \mathrm{R}=-\mathrm{OCF}_{3}$

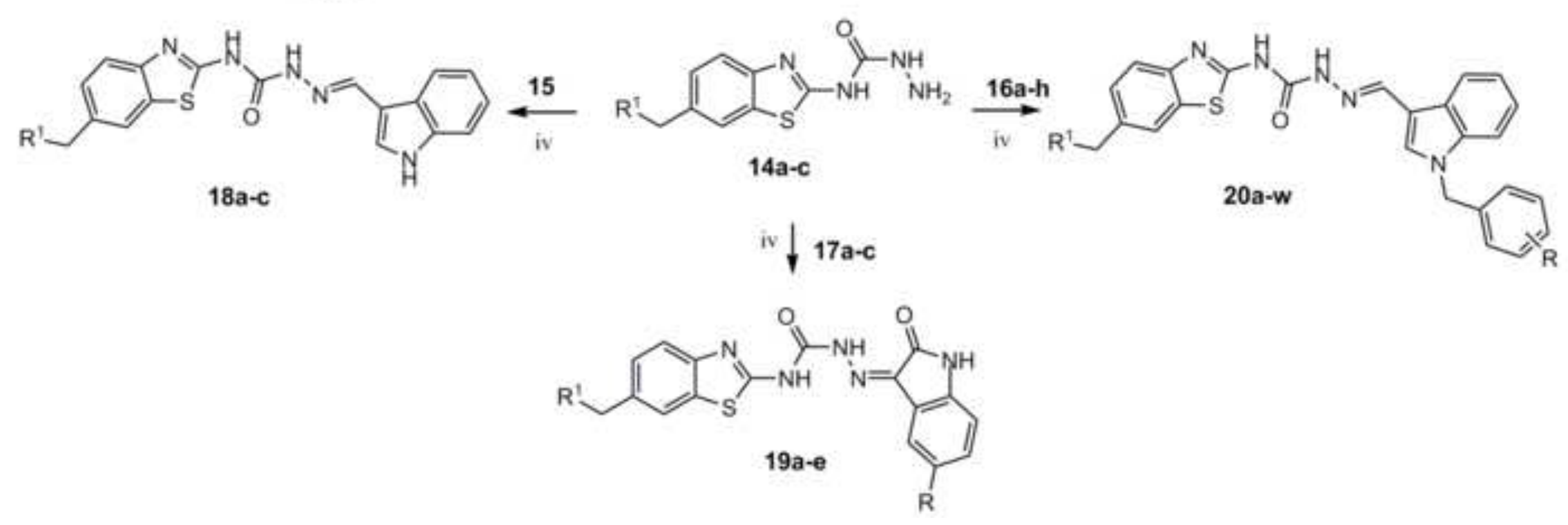




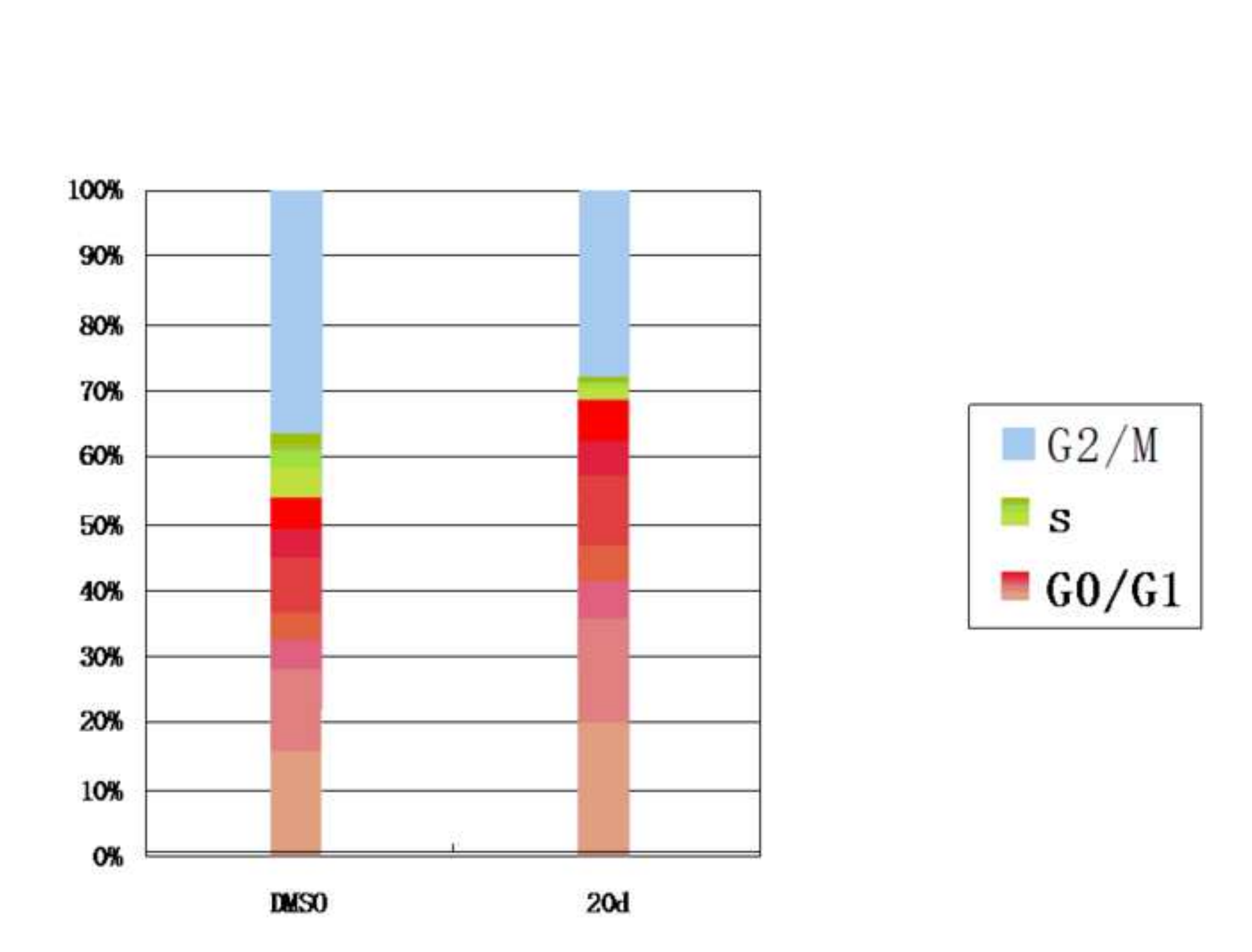

G2/M

S

믈 $0 / \mathrm{G} 1$

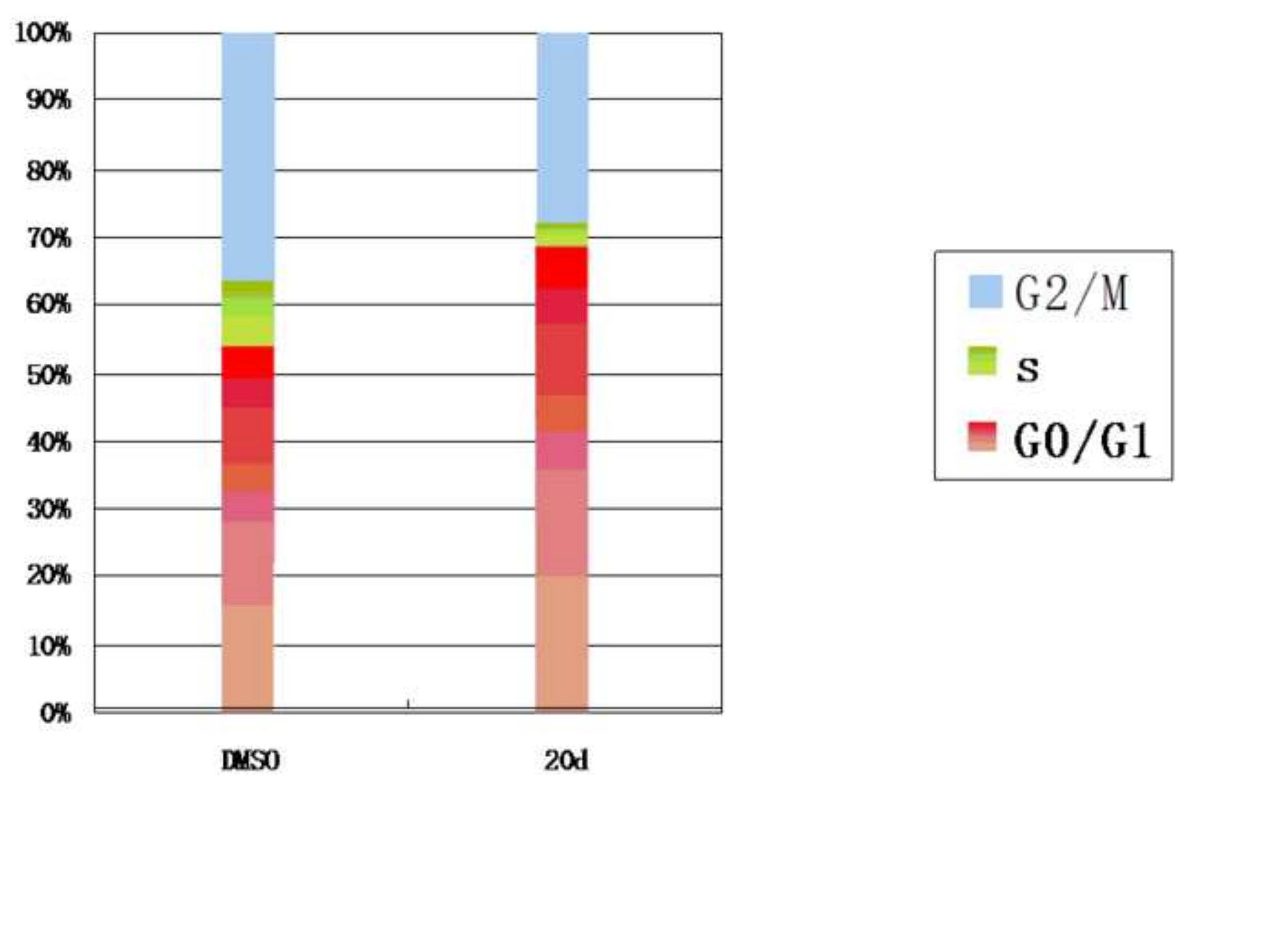

(1)

(1)

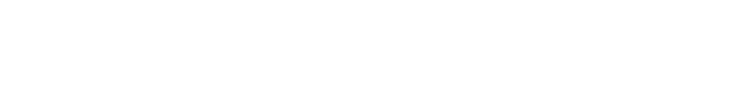

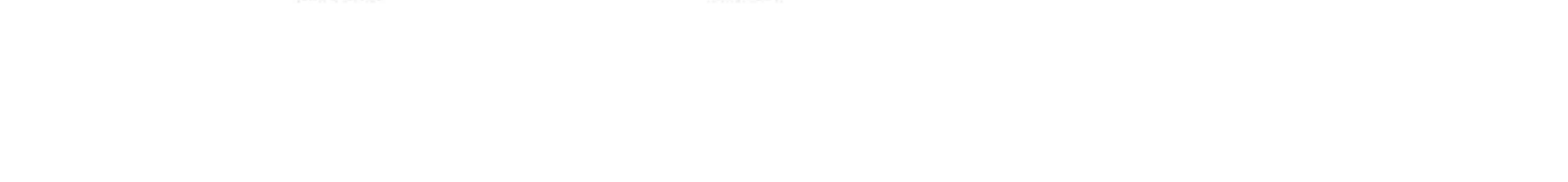

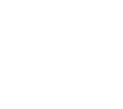

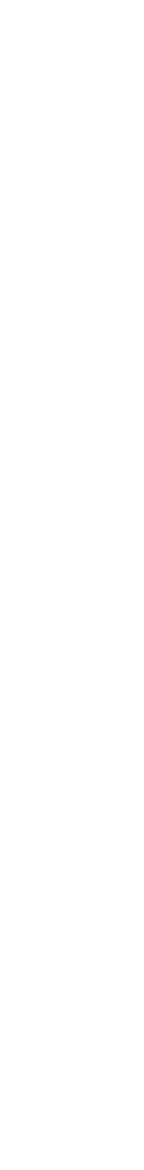

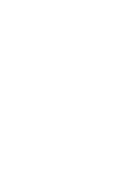




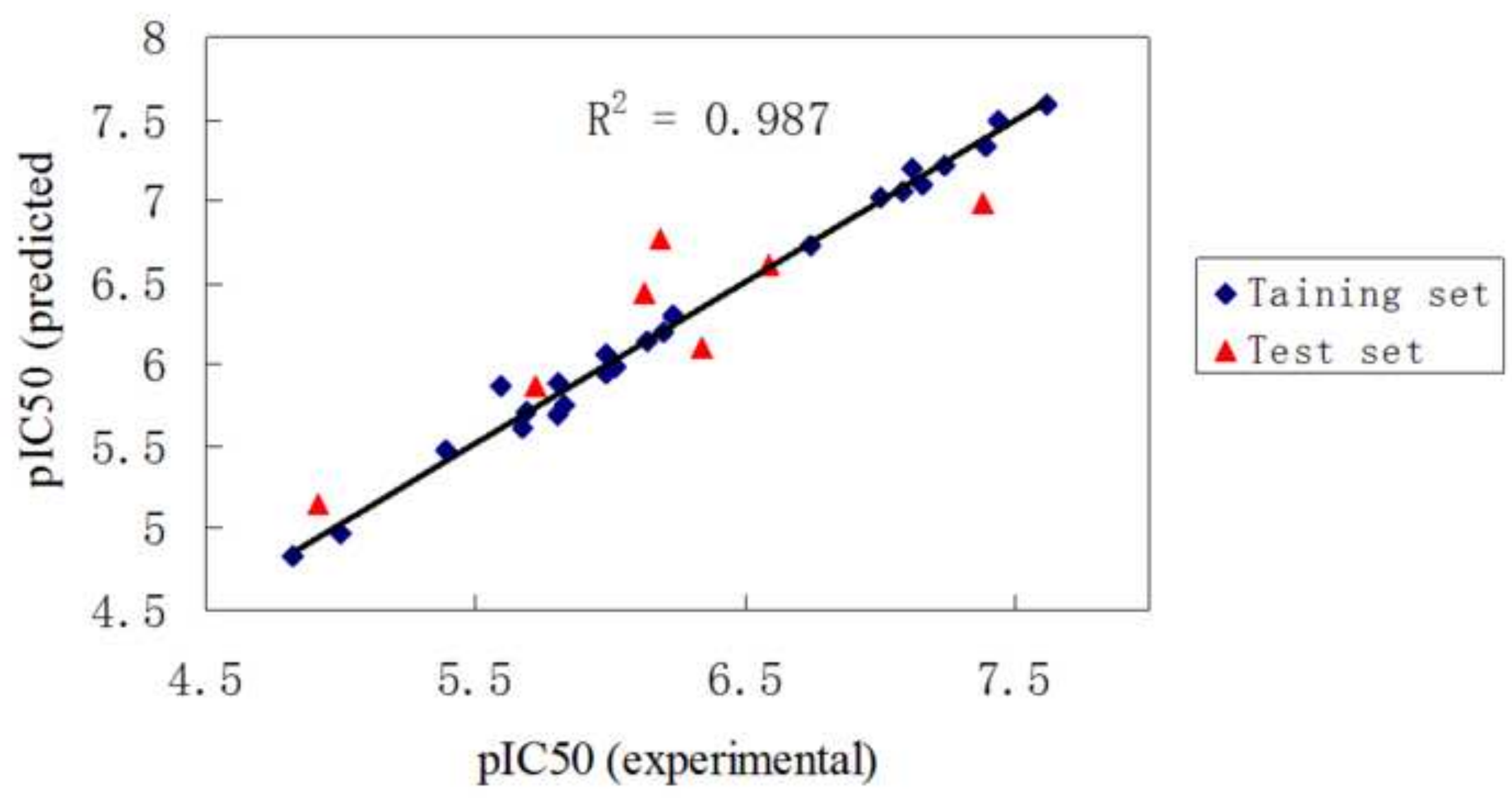

- Taining set

$\Delta$ Test set 

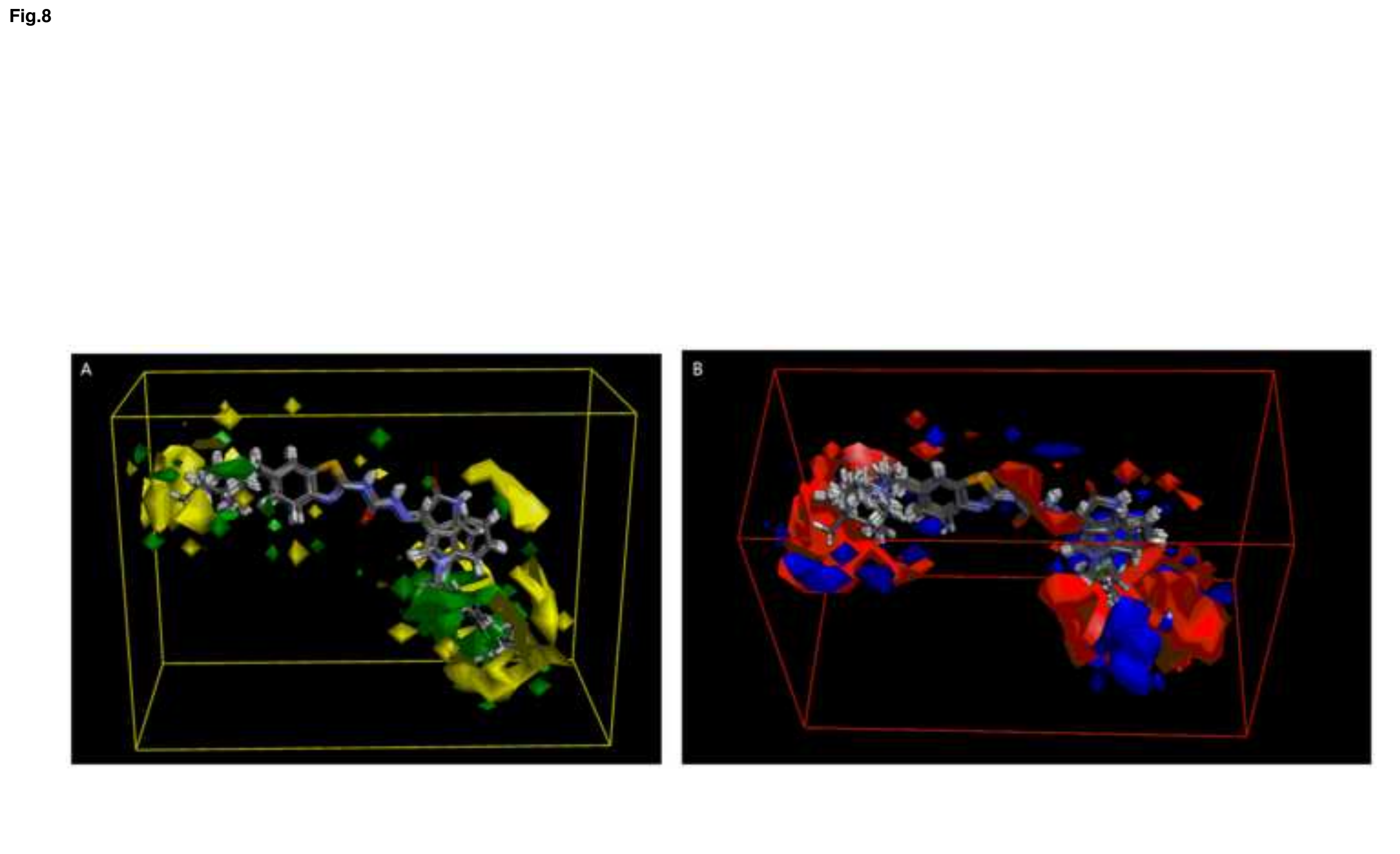

.

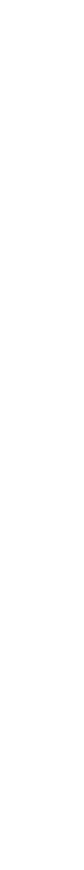

